\title{
The fourth moment of derivatives of Dirichlet $L$-functions in function fields
}

\author{
Julio Cesar Andrade ${ }^{1} \cdot$ Michael Yiasemides $^{1}$
}

Received: 4 July 2019 / Accepted: 14 November 2020 / Published online: 26 February 2021

(c) The Author(s) 2021

\begin{abstract}
We obtain the asymptotic main term of moments of arbitrary derivatives of $L$-functions in the function field setting. Specifically, we obtain the first, second, and mixed fourth moments. The average is taken over all non-trivial characters of a prime modulus $Q \in \mathbb{F}_{q}[T]$, and the asymptotic limit is as $\operatorname{deg} Q \longrightarrow \infty$. This extends the work of Tamam who obtained the asymptotic main term of low moments of $L$-functions, without derivatives, in the function field setting. It is also the function field $q$-analogue of the work of Conrey, who obtained the fourth moment of derivatives of the Riemann zeta-function.
\end{abstract}

Keywords Moments of $L$-functions · Dirichlet character · Polynomial · Function field · Derivative

Mathematics Subject Classification Primary 11M06; Secondary 11M38 $\cdot$ 11M50 $\cdot 11$ N36

\section{Contents}

1 Introduction . . . . . . . . . . . . . . . . . . . . . . . 672

2 Notation and statement of results . . . . . . . . . . . . . . . . . . . . . . . . . . . . . 674

3 Preliminary results . . . . . . . . . . . . . . . . . . . . . . . . . 677

4 First moments . . . . . . . . . . . . . . . . . . . . . . . . . . . . 679

5 Second moments . . . . . . . . . . . . . . . . . . . . . . . . . . . . . 680

6 Fourth moments: expressing as manageable summations . . . . . . . . . . . . . . . . . . . . . 6 681

7 Fourth moments: handling the summations . . . . . . . . . . . . . . . . . . . . . . . 687

8 Fourth moments of derivatives . . . . . . . . . . . . . . . . . . . . . . . . . . . . 692

References ................................ 696

Michael Yiasemides my298@exeter.ac.uk

Julio Cesar Andrade

j.c.andrade@exeter.ac.uk

1 Department of Mathematics, University of Exeter, Exeter EX4 4QF, UK 


\section{Introduction}

The moments of families of $L$-functions are part of an important area of research in analytic number theory. The moments of the Riemann zeta-function, averaged over the critical line, have applications to areas such as zero density estimates and the proportion of zeros on the critical line [12]. Furthermore, the Lindelöf hypothesis can be expressed in terms of such moments. One can also study moments over families of $L$-functions that are evaluated at the central point, which has applications to the non-vanishing of $L$-functions at the central point.

In 1916, Hardy and Littlewood [13] proved that

$$
\frac{1}{T} \int_{0}^{T}\left|\zeta\left(\frac{1}{2}+i t\right)\right|^{2} \mathrm{~d} t \sim \log T
$$

as $T \longrightarrow \infty$. In 1926, Ingham [16] expanded on this by proving that

$$
\frac{1}{T} \int_{0}^{T}\left|\zeta\left(\frac{1}{2}+i t\right)\right|^{4} \mathrm{~d} t \sim \frac{1}{2 \pi} \log ^{4} T
$$

as $T \longrightarrow \infty$. In 1979, Heath-Brown [14] obtained lower order terms for the fourth moment above. Results for moments of powers greater than 4 have resisted the attempts of mathematicians for many years. Indeed, at this time, we can only conjecture such results. In 2000, Keating and Snaith [18] conjectured, using random matrix theory, the asymptotic main term for all even moments:

$$
\frac{1}{T} \int_{t=0}^{T}\left|\zeta\left(\frac{1}{2}+i t\right)\right|^{2 k} \mathrm{~d} t \sim a_{k} \frac{G^{2}(1+k)}{G(1+2 k)}(\log T)^{k^{2}}
$$

as $\operatorname{deg} T \longrightarrow \infty$, where

$$
a_{k}:=\prod_{p \text { prime }}\left(1-\frac{1}{p}\right)^{k^{2}} \sum_{m=0}^{\infty} \frac{d_{k}\left(p^{m}\right)^{2}}{p^{m}},
$$

$d_{k}(a)$ is the number of ways of expressing $a$ as a product of $k$ factors, and $G$ is the Barnes $G$-function. The factor of $a_{k}$ above does not occur in a natural way via the method in [18]. Indeed, random matrix theory allows us to conjecture the factor $\frac{G^{2}(1+k)}{G(1+2 k)}$, and the fact that we must then include the factor $a_{k}$ can be seen from other results. However, this was addressed by Gonek, Hughes, and Keating [11] where they developed a method for conjecturing the main term of the even moments of $\zeta(s)$ in such a way that all factors appear naturally. They give an Euler-Hadamard hybrid formula for the $\zeta(s)$. That is, they express $\zeta(s)$ as, roughly, a partial product over primes multiplied by a partial product over the zeros of $\zeta(s)$. The former contributes the factor $a_{k}$, while random matrix theory is used to conjecture that the latter contributes the factor $\frac{G^{2}(1+k)}{G(1+2 k)}$.

With regards to moments over families of $L$-functions, we briefly consider the family of Dirichlet $L$-functions, as that is the focus of this paper. Paley [22] is accredited to proving that

$$
\frac{1}{\phi^{*}(q)} \sum_{\chi \bmod q}^{*}\left|L\left(\frac{1}{2}, \chi\right)\right|^{2} \sim \frac{\phi(q)}{q} \log q
$$

as $q \longrightarrow \infty$. Here, the sum is over all primitive characters of modulus $q ; \phi^{*}(q)$ is the number of primitive characters of modulus $q$; $\phi$ is the totient function; and $L(s, \chi)$ is the 
Dirichlet $L$-function associated to the character $\chi$. Extending the work of Heath-Brown [15], Soundararajan [24] proved that

$$
\frac{1}{\phi^{*}(q)} \sum_{\chi \bmod q}^{*}\left|L\left(\frac{1}{2}, \chi\right)\right|^{4} \sim \frac{1}{2 \pi^{2}} \prod_{p \mid q} \frac{\left(1-p^{-1}\right)^{3}}{1+p^{-1}}(\log q)^{4} .
$$

More recently, Young [26] obtained lower order terms for the fourth moment above. By adapting the method of [11], Bui and Keating [6] conjectured the main term of all even moments of Dirichlet $L$-functions at $\frac{1}{2}$ :

$$
\frac{1}{\phi^{*}(q)} \sum_{\chi \bmod q}^{*}\left|L\left(\frac{1}{2}, \chi\right)\right|^{2 k} \sim a_{k} \frac{G^{2}(1+k)}{G(1+2 k)} \prod_{p \mid q}\left(\sum_{m=0}^{\infty} \frac{d_{k}\left(p^{m}\right)^{2}}{p^{m}}\right)^{-1}(\log q)^{k^{2}} .
$$

We note a similarity between the above and (1). The reason for this is that the family of Dirichlet $L$-functions and the Riemann zeta function share the same symmetry [7, Section 1], which we briefly touch upon later.

We must also remark that derivatives of $L$-functions are an important area of study. The derivative of $\zeta(s)$ plays a key role in obtaining positive lower bounds for the proportion of non-trivial zeros of $\zeta(s)$ on the critical line [4,9,20]. Furthermore, discrete moments of $\zeta^{\prime}(s)$, where one averages over the zeros of $\zeta(s)$, can be used to understand the number of simple zeros of $\zeta(s)$ on the critical line (see [12] for a brief explanation of this and other related results). The derivatives of Dirichlet $L$-functions are of interest because this family of $L$-functions shares the same symmetry as the Riemann zeta function, and so there are analogies between their derivatives as well. Moments of derivatives of Dirichlet $L$-functions at central point of $\frac{1}{2}$ have applications to the non-vanishing of these derivatives at that point. More generally, for some results on the moments of derivatives of automorphic $L$-functions and applications, we refer the reader to [21]. We now reference two results that we require for comparisons that we will make later. Conrey, Rubinstein, and Snaith [10] conjectured, using random matrix theory, that, for positive integers $k$,

$$
\frac{1}{T} \int_{t=0}^{T}\left|\zeta^{\prime}\left(\frac{1}{2}+i t\right)\right|^{2 k} \mathrm{~d} t \sim a_{k} b_{k}(\log T)^{k^{2}+2 k}
$$

as $\operatorname{deg} T \longrightarrow \infty$, where $a_{k}$ is as in (2) and values for $b_{1}, b_{2}, \ldots, b_{15}$ are explicitly given. In particular,

$$
b_{1}=\frac{1}{3}, \quad b_{2}=\frac{61}{2^{5} \cdot 3^{2} \cdot 5 \cdot 7} .
$$

This is consistent with a rigorously established result of Conrey [8]:

$$
\frac{1}{T} \int_{t=0}^{T}\left|\zeta^{\prime}\left(\frac{1}{2}+i t\right)\right|^{4} \mathrm{~d} t \sim a_{k} \frac{61}{2^{5} \cdot 3^{2} \cdot 5 \cdot 7}(\log T)^{8} .
$$

Conrey also showed that

$$
\frac{\pi^{2}}{6} C_{2, m} \sim \frac{1}{16 m^{4}}
$$

as $m \longrightarrow \infty$, where

$$
C_{2, m}=\lim _{T \longrightarrow \infty} T^{-1}\left(\log \left(\frac{T}{2 \pi}\right)\right)^{-4 m-4} \int_{t=1}^{T}\left|\zeta^{(m)}\left(\frac{1}{2}+i t\right)\right|^{4} \mathrm{~d} t .
$$


There is another setting in which one can study $L$-functions: The function field setting. Here, our $L$-functions are defined as sums over monic polynomials from an appropriate polynomial ring. For details and notation, see Sect. 2. There are many analogies between the classical setting and the function field setting, including an analogue of the Riemann hypothesis (one of the conjectures of Weil), which has been proved (first by Deligne). Lfunctions in function fields also play a crucial role in our understanding of the symmetries of families of $L$-functions in both the classical and function field settings. Indeed, for families of classical $L$-functions, one must consider their function field analogue, for which we can obtain indications as to their symmetry because, in this setting, we have a spectral interpretation of the zeros of the $L$-functions. For details, we refer the reader to the work of Katz and Sarnak [17], as well as Conrey and Farmer [7].

We now give a brief description of some of the results regarding moments of $L$-functions in the function field setting. Tamam [25] obtained a special case of the function field analogue of (3):

$$
\frac{1}{\phi(Q)} \sum_{\chi \bmod Q}^{*}\left|L\left(\frac{1}{2}, \chi\right)\right|^{4}=\frac{1-q^{-1}}{12}(\operatorname{deg} Q)^{4}+O\left((\operatorname{deg} Q)^{3}\right)
$$

as $\operatorname{deg} Q \longrightarrow \infty$ with $Q$ being prime. Andrade and Yiasemides [3] generalised this to the full analogue of Soundararajan's result, by removing the restriction that $Q$ be prime. As we will soon see, this current paper considers another generalisation of Tamam's result. Namely, we still impose that $Q$ is prime, but we consider arbitrary derivatives of the $L$-functions instead of the $L$-function itself. A function field analogue of [6] can be found in the thesis of Yiasemides (yet to be published), where conjectures of all even moments of Dirichlet $L$-functions in function fields are given, as well as an extension of this to the first derivatives of the $L$-functions. With regards to moments of the family of quadratic Dirichlet $L$-functions in function fields, we refer the reader to the work of Andrade and Keating [1,2], and to the work of Bui and Florea [5] for an approach to conjecturing higher moments via the method of the Euler-Hadamard hybrid formula.

These are but a few of the many results regarding moments of $L$-functions in function fields. For an introduction to number theory in function fields, we refer the reader to the book by Rosen [23].

\section{Notation and statement of results}

Henceforth, $q$ will represent an integer prime power, not equal to 2 . For all such $q$ we have a finite field of order $q$, denoted by $\mathbb{F}_{q}$. The polynomial ring over this finite field is denoted by $\mathbb{F}_{q}[T]$, but, as we are working with a general prime power $q \neq 2$, we will simply write $\mathcal{A}$ for $\mathbb{F}_{q}[T]$. The subset of monic polynomials is denoted by $\mathcal{M}$. The degree of a polynomial is the standard definition, although we do not define it for the zero polynomial. Hence, the range $\operatorname{deg} A \leq n$, for any non-negative integer $n$, does not include the case $A=0$. For $A \in \mathcal{A} \backslash\{0\}$ we define the norm of $A$ as $|A|:=q^{\operatorname{deg} A}$, and for $A=0$ we define $|0|:=0$.

Generally, we reserve upper-case letters for elements of $\mathcal{A}$, and the letters $P$ and $Q$ are reserved for prime polynomials. Note that primality and irreducibility are equivalent as $\mathcal{A}$ is a Euclidean domain. In this paper, the term "prime" is taken to mean "monic prime". We denote the set of monic primes in $\mathcal{A}$ by $\mathcal{P}$. The expression $\operatorname{deg} Q \longrightarrow \infty$ should be taken to mean "deg $Q \longrightarrow \infty$ with $Q$ being prime". For $A, B \in \mathcal{A}$ we denote the highest common factor and lowest common multiple by $(A, B)$ and $[A, B]$, respectively. 
For a subset $\mathcal{S} \subseteq \mathcal{A}$ we define, for all non-negative integers $n, \mathcal{S}_{n}:=\{A \in \mathcal{S}: \operatorname{deg} A=n\}$. We identify $\mathcal{A}_{0}$ with $\mathbb{F}_{q} \backslash\{0\}=\mathbb{F}_{q}{ }^{*}$. For a $k$-times differentiable function $f(x)$, we defined $f^{(k)}(x)$ to be its $k$-th derivative.

We use the "big $O$ " and "little $o$ " notation, and subscripts demonstrate the dependencies of the implied constant. That is, suppose we have functions $f(x)$ and $g(x)$ with the same domain $D$. Then, we write $f(x)=O(g(x))$ or $f(x) \ll g(x)$ if and only if there is exists some positive constant $c$ such that $|f(x)| \leq c|g(x)|$ for all $x \in D$. If, instead, we have functions $f_{k}(x)$ and $g_{k}(x)$ that depend on some parameter $k$, then we write $f_{k}(x)=O\left(g_{k}(x)\right)$ or $f_{k}(x) \ll g_{k}(x)$ if and only if there exists some positive constant $c$ such that for all $k$ and all $x \in D$ we have $\left|f_{k}(x)\right| \leq c\left|g_{k}(x)\right|$. Also, if only $f_{k}(x)$ is dependent on $k$, and $g(x)$ is not, then we write $f_{k}(x)=O_{k}(g(x))$ or $f_{k}(x) \ll_{k} g(x)$ if and only if for all $k$ there exists some positive constant $c_{k}$ such that for all $x \in D$ we have $\left|f_{k}(x)\right| \leq c_{k}|g(x)|$. Usually, the parameter that our functions may depend on will be the prime power $q$, and our implied constant will in fact be independent of $q$. The expression " $f(x) \sim g(x)$ as $x \longrightarrow \infty$ " is taken to mean $\lim _{x \rightarrow \infty} \frac{f(x)}{g(x)}=1$.

Definition 2.1 (Dirichlet character) Let $R \in \mathcal{M}$. A Dirichlet character on $\mathcal{A}$ of modulus $R$ is a function $\chi: \mathcal{A} \longrightarrow \mathbb{C}^{*}$ satisfying the following properties for all $A, B \in \mathcal{A}$ :

(1) $\chi(A)=\chi(B)$ if $A \equiv B(\bmod R)$;

(2) $\chi(A B)=\chi(A) \chi(B)$;

(3) $\chi(A)=0$ if and only if $(A, R) \neq 1$.

We denote a sum over all characters of modulus $R$ by $\sum_{\chi \bmod R}$. Also, note that we can view $\chi$ as a function on $\mathcal{A} / R \mathcal{A}$, which follows naturally from point (1) above. This will allow us to use expressions such as $\chi\left(A^{-1}\right)$ when $A \in(\mathcal{A} / R \mathcal{A})^{*}$.

We say $\chi$ is the trivial character of modulus $R$ if $\chi(A)=1$ for all $A \in \mathcal{A}$ satisfying $(A, R)=1$, and we denote such characters by $\chi_{0}$ (the dependence on the modulus $R$ is not shown with this notation, but when used it will be clear what the associated modulus is). We define the even characters to be those characters $\chi$ satisfying $\chi(a)=1$ for all $a \in \mathbb{F}_{q}^{*}$. Otherwise, we say that the character is odd. It can be shown that there are $\phi(R)$ characters of modulus $R$ and $\frac{\phi(R)}{q-1}$ even characters of modulus $R$, where $\phi$ is the totient function. This follows from the fact that a finite abelian group is isomorphic to its dual. Specifically, we refer the reader to Theorem 9.1 and Corollary 9.3 of [19], where one should take the abelian group in the theorem to be $(\mathcal{A} / R \mathcal{A})^{*}$ and the subgroup in the corollary to be $\mathbb{F}_{q}^{*}$.

Now, suppose $\chi$ is a character of modulus $R \in \mathcal{M}$ and further suppose that $S \mid R$. We say that $S$ is an induced modulus of $\chi$ if there exists a character $\chi_{1}$ of modulus $S$ such that

$$
\chi(A)= \begin{cases}\chi_{1}(A) & \text { if }(A, R)=1 \\ 0 & \text { otherwise. }\end{cases}
$$

We say that $\chi$ is primitive if there is no induced modulus of strictly smaller degree than $R$. Otherwise, $\chi$ is said to be non-primitive. We denote the number of primitive characters of modulus $R$ by $\phi^{*}(R)$. We note that all trivial characters of modulus $R \in \mathcal{M} \backslash\{1\}$ are nonprimitive as they are induced by the character of modulus 1 . We also note that if $Q$ is prime, then the only non-primitive character of modulus $Q$ is the trivial character of modulus $Q$. In particular, if $Q$ is prime then $\phi^{*}(Q)=\phi(Q)-1 \sim \phi(Q)$ as $\operatorname{deg} Q \longrightarrow \infty$. We denote a sum over all primitive characters $\chi$ of modulus $R$ by $\sum_{\chi \bmod R}^{*}$, and if $Q$ is prime then it is equivalent to write $\sum_{\substack{\chi \bmod _{\chi} \\ \chi \neq \chi_{0}}}$. 
Definition 2.2 (Dirichlet $L$-function) Let $\chi$ be a Dirichlet character of modulus $R$. We define the associated Dirichlet $L$-function as follows: For all $\operatorname{Re}(s)>1$,

$$
L(s, \chi):=\sum_{A \in \mathcal{M}} \frac{\chi(A)}{|A|^{s}}=\sum_{n=0}^{\infty} L_{n}(\chi)\left(q^{-s}\right)^{n},
$$

where

$$
L_{n}(\chi):=\sum_{\substack{A \in \mathcal{M} \\ \operatorname{deg} A=n}} \chi(A)
$$

This has an analytic continuation to $\mathbb{C}$ for non-trivial characters, and to $\mathbb{C} \backslash\left\{1+\frac{2 n \pi i}{\log q}: n \in \mathbb{Z}\right\}$ for trivial characters.

Definition 2.3 (Riemann Zeta-function in $\mathbb{F}_{q}[T]$ ) When $\chi$ is the Dirichlet character of modulus 1 , then the associated Dirichlet $L$-function is simply the Riemann zeta-function on $\mathcal{A}$, namely

$$
\zeta_{A}(s):=\sum_{A \in \mathcal{M}} \frac{1}{|A|^{s}}
$$

We now state the main results of this paper.

Theorem 2.4 For all positive integers $k$, we have that

$$
\frac{1}{\phi(Q)} \sum_{\substack{\bmod _{\chi \neq \chi_{0}} \\ \chi L^{(k)}}}\left(\frac{1}{2}, \chi\right)=\frac{-(-\log q)^{k}}{q^{\frac{1}{2}}-1} \frac{(\operatorname{deg} Q)^{k}}{|Q|^{\frac{1}{2}}}+O_{k}\left((\log q)^{k} \frac{(\operatorname{deg} Q)^{k-1}}{|Q|^{\frac{1}{2}}}\right) .
$$

Theorem 2.5 For all positive integers $k$ we have that

$$
\frac{1}{\phi(Q)} \sum_{\substack{\chi \bmod Q \\ \chi \neq \chi_{0}}}\left|L^{(k)}\left(\frac{1}{2}, \chi\right)\right|^{2}=\frac{(\log q)^{2 k}}{2 k+1}(\operatorname{deg} Q)^{2 k+1}+O\left((\log q)^{2 k}(\operatorname{deg} Q)^{2 k}\right) .
$$

Theorem 2.6 For all non-negative integers $k, l$ we have that

$$
\begin{aligned}
& \frac{1}{\phi(Q)} \frac{1}{(\log q)^{2 k+2 l}} \sum_{\substack{\chi \bmod _{\chi \neq} Q \\
\chi \neq \chi_{0}}}\left|L^{(k)}\left(\frac{1}{2}, \chi\right)\right|^{2}\left|L^{(l)}\left(\frac{1}{2}, \chi\right)\right|^{2} \\
= & \left(1-q^{-1}\right)(\operatorname{deg} Q)^{2 k+2 l+4} \\
& \times \int_{\substack{a_{1}, a_{2}, a_{3}, a_{4} \geq 0 \\
2 a_{1}+a_{3}+a_{4}<1 \\
2 a_{2}+a_{3}+a_{4}<1}} f_{k}\left(a_{1}+a_{3}, a_{1}+a_{4}, 1\right) f_{l}\left(a_{2}+a_{4}, a_{2}+a_{3}, 1\right) \mathrm{d} a_{1} \mathrm{~d} a_{2} \mathrm{~d} a_{3} \mathrm{~d} a_{4} \\
& +O_{k, l}\left((\operatorname{deg} Q)^{2 k+2 l+\frac{7}{2}}\right),
\end{aligned}
$$

where for all non-negative integers $i$ we define

$$
f_{i}(x, y, z)=x^{i} y^{i}+(z-x)^{i}(z-y)^{i} .
$$


Notice the similarity between the conjecture (4) for $k=1,2$ and Conrey's result (5), and the corresponding special cases of our results:

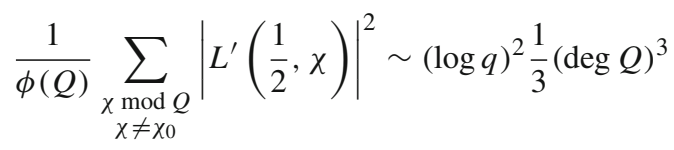

and

$$
\frac{1}{\phi(Q)} \sum_{\substack{\chi \bmod _{\chi} Q \\ \chi \neq \chi_{0}}}\left|L^{\prime}\left(\frac{1}{2}, \chi\right)\right|^{4} \sim(\log q)^{4}\left(1-q^{-1}\right) \frac{61}{2^{5} \cdot 3^{2} \cdot 5 \cdot 7}(\operatorname{deg} Q)^{8} .
$$

This is not surprising given that the Riemann zeta-function and the family of Dirichlet $L$ functions share the same symmetry, as mentioned previously.

We also prove the following result:

Theorem 2.7 For all non-negative integers $m$ we define

$$
\begin{aligned}
D_{m}:= & \lim _{\operatorname{deg} Q \rightarrow \infty} \frac{1}{\left(1-q^{-1}\right)(\log q)^{4 m}} \frac{1}{\phi(Q)} \frac{1}{(\operatorname{deg} Q)^{4 m+4}} \sum_{\substack{\chi \bmod _{\chi \neq \chi} \\
\chi \neq \chi_{0}}}\left|L^{(m)}\left(\frac{1}{2}, \chi\right)\right|^{4} \\
= & \int_{\substack{a_{1}, a_{2}, a_{3}, a_{4} \geq 0 \\
2 a_{1}+a_{3}+a_{4}<1 \\
2 a_{2}+a_{3}+a_{4}<1}}\left(\left(a_{1}+a_{3}\right)^{m}\left(a_{1}+a_{4}\right)^{m}+\left(1-a_{1}-a_{3}\right)^{m}\left(1-a_{1}-a_{4}\right)^{m}\right) \\
& \times\left(\left(a_{2}+a_{3}\right)^{m}\left(a_{2}+a_{4}\right)^{m}+\left(1-a_{2}-a_{3}\right)^{m}\left(1-a_{2}-a_{4}\right)^{m}\right) \mathrm{d} a_{1} \mathrm{~d} a_{2} \mathrm{~d} a_{3} \mathrm{~d} a_{4} .
\end{aligned}
$$

We have that

$$
D_{m} \sim \frac{1}{16 m^{4}}
$$

as $m \longrightarrow \infty$.

We note the similarity between our result and (6). Note that the factor of $\zeta(2)=\frac{\pi^{2}}{6}$ in (6) corresponds to the factor of $\zeta_{\mathcal{A}}(2)=\frac{1}{1-q^{-1}}$ in our definition of $D_{m}$.

\section{Preliminary results}

The following results are well known and, for many, the proofs can be found in Rosen's book [23].

Lemma 3.1 Let $\chi$ be a non-trivial Dirichlet character modulo $R \in \mathcal{M}$. Then,

$$
\sum_{A \in \mathcal{A} / R \mathcal{A}} \chi(A)=\sum_{A \in(\mathcal{A} / R \mathcal{A})^{*}} \chi(A)=0 .
$$

Lemma 3.2 Let $\chi$ be an odd Dirichlet character. Then,

$$
\sum_{a \in \mathbb{F}_{q}} \chi(a)=\sum_{a \in \mathbb{F}_{q}^{*}} \chi(a)=0
$$


Lemma 3.3 Let $R \in \mathcal{M}$. Then,

$$
\sum_{\chi \bmod R} \chi(A)= \begin{cases}\phi(R) & \text { if } A \equiv 1(\bmod R) \\ 0 & \text { otherwise }\end{cases}
$$

and, if we also have $R \neq 1$,

$$
\sum_{\substack{\chi \bmod R \\ \chi \text { even }}} \chi(A)= \begin{cases}\frac{\phi(R)}{q-1} & \text { if } A \equiv a(\bmod R) \text { for some } a \in\left(\mathbb{F}_{q}\right)^{*} \\ 0 & \text { otherwise. }\end{cases}
$$

Corollary 3.4 Let $R \in \mathcal{M}$. Then,

$$
\sum_{\chi \bmod R} \chi(A) \bar{\chi}(B)= \begin{cases}\phi(R) & \text { if }(A B, R)=1 \text { and } A \equiv B(\bmod R) \\ 0 & \text { otherwise, }\end{cases}
$$

and, if we also have $R \neq 1$,

$$
\sum_{\substack{\chi \text { moden } \\ \chi \text { even }}} \chi(A) \bar{\chi}(B)= \begin{cases}\frac{\phi(R)}{q-1} & \text { if }(A B, R)=1 \text { and } A \equiv a B(\bmod R) \text { for some } a \in\left(\mathbb{F}_{q}\right)^{*} \\ 0 & \text { otherwise. }\end{cases}
$$

Lemma 3.5 We have that

$$
\zeta_{\mathcal{A}}(s)=\frac{1}{1-q^{1-s}}
$$

and this gives an analytic continuation of $\zeta_{\mathcal{A}}$ to $\mathbb{C} \backslash\left\{1+\frac{2 n \pi i}{\log q}: n \in \mathbb{Z}\right\}$. We also have the following Euler product for $\operatorname{Re}(s)>1$ :

$$
\zeta_{\mathcal{A}}(s)=\prod_{P \in \mathcal{P}}\left(1-|P|^{-s}\right)^{-1} .
$$

Lemma 3.5 can be generalised to the following:

Lemma 3.6 Let $R \in \mathcal{M}$ and let $\chi$ be a Dirichlet character of modulus $R$. If $\chi=\chi_{0}$ then we have

$$
L\left(s, \chi_{0}\right)=\left(\prod_{P \mid R} 1+|P|^{-s}\right) \zeta_{\mathcal{A}}(s)
$$

If $\chi \neq \chi_{0}$ then we have

$$
L(s, \chi)=\sum_{\substack{A \in \mathcal{M} \\ \operatorname{deg} A<\operatorname{deg} R}} \frac{\chi(A)}{|A|^{s}} .
$$

We can now see how the analytic continuations given in the introduction are obtained.

Lemma 3.7 For $\operatorname{Re}(r), \operatorname{Re}(s)>1$, we have that

$$
\sum_{\substack{R, S \in \mathcal{M} \\(R, S)=1}} \frac{1}{|R|^{r}|S|^{s}}=\left(\sum_{R \in \mathcal{M}} \frac{1}{|R|^{r}}\right)\left(\sum_{S \in \mathcal{M}} \frac{1}{|S|^{s}}\right)\left(1-q^{1-r-s}\right) .
$$


Proof We have that

$$
\begin{aligned}
\sum_{R, S \in \mathcal{M}} \frac{1}{|R|^{r}|S|^{s}} & =\sum_{G \in \mathcal{M}} \sum_{\substack{R, S \in \mathcal{M} \\
(R, S)=G}} \frac{1}{|R|^{r}|S|^{s}}=\sum_{G \in \mathcal{M}} \frac{1}{|G|^{r}|G|^{s}} \sum_{\substack{R, S \in \mathcal{M} \\
(R, S)=1}} \frac{1}{|R|^{r}|S|^{s}} \\
& =\left(\sum_{G \in \mathcal{M}} \frac{1}{|G|^{r+s}}\right)\left(\sum_{\substack{R, S \in \mathcal{M} \\
(R, S)=1}} \frac{1}{|R|^{r}|S|^{s}}\right)
\end{aligned}
$$

From this we easily deduce that

$$
\begin{aligned}
\sum_{\substack{R, S \in \mathcal{M} \\
(R, S)=1}} \frac{1}{|R|^{r}|S|^{s}} & =\left(\sum_{R, S \in \mathcal{M}} \frac{1}{|R|^{r}|S|^{s}}\right)\left(\sum_{G \in \mathcal{M}} \frac{1}{|G|^{r+s}}\right)^{-1} \\
& =\left(\sum_{R \in \mathcal{M}} \frac{1}{|R|^{r}}\right)\left(\sum_{S \in \mathcal{M}} \frac{1}{|S|^{s}}\right)\left(1-q^{1-r-s}\right) .
\end{aligned}
$$

\section{First moments}

To prove Theorem 2.4 we will require the following lemma.

Lemma 4.1 For all positive integers $k$ we have that

$$
\sum_{n=0}^{\operatorname{deg} Q-1} n^{k} q^{\frac{n}{2}}=\frac{1}{q^{\frac{1}{2}}-1}(\operatorname{deg} Q)^{k}|Q|^{\frac{1}{2}}+O_{k}\left((\operatorname{deg} Q)^{k-1}|Q|^{\frac{1}{2}}\right) .
$$

Proof We have that

$$
\begin{aligned}
\sum_{n=0}^{\operatorname{deg} Q-1} n^{k} q^{\frac{n}{2}} & =\frac{1}{q^{\frac{1}{2}}-1} \sum_{n=0}^{\operatorname{deg} Q-1}\left(n^{k} q^{\frac{n+1}{2}}-n^{k} q^{\frac{n}{2}}\right) \\
& =\frac{1}{q^{\frac{1}{2}}-1} \sum_{n=0}^{\operatorname{deg} Q-1}\left((n+1)^{k} q^{\frac{n+1}{2}}-n^{k} q^{\frac{n}{2}}\right) \\
& -\frac{1}{q^{\frac{1}{2}}-1} \sum_{n=0}^{\operatorname{deg} Q-1}\left((n+1)^{k} q^{\frac{n+1}{2}}-n^{k} q^{\frac{n+1}{2}}\right) \\
= & \frac{1}{q^{\frac{1}{2}}-1}(\operatorname{deg} Q)^{k}|Q|^{\frac{1}{2}}+O\left(\sum_{i=0}^{k-1}\left(\begin{array}{l}
k \\
i
\end{array}\right)(\operatorname{deg} Q)^{i} \sum_{n=0}^{\operatorname{deg} Q-1} q^{\frac{n+1}{2}}\right) \\
= & \frac{1}{q^{\frac{1}{2}}-1}(\operatorname{deg} Q)^{k}|Q|^{\frac{1}{2}}+O_{k}\left((\operatorname{deg} Q)^{k-1}|Q|^{\frac{1}{2}}\right)
\end{aligned}
$$


Proof of Theorem 2.4 We can easily see that

$$
L^{(k)}(s, \chi)=(-\log q)^{k} \sum_{n=1}^{\operatorname{deg} Q-1} n^{k} q^{-n s} \sum_{\substack{A \in \mathcal{M} \\ \operatorname{deg} A=n}} \chi(A),
$$

from which we deduce that

$$
\begin{aligned}
\frac{1}{\phi(Q)} \sum_{\substack{\bmod _{\chi \neq} \\
\chi \neq \chi_{0}}} L^{(k)}\left(\frac{1}{2}, \chi\right) & =\frac{(-\log q)^{k}}{\phi(Q)} \sum_{n=1}^{\operatorname{deg} Q-1} n^{k} q^{-\frac{n}{2}} \sum_{\substack{A \in \mathcal{M} \\
\operatorname{deg} A=n}} \sum_{\substack{\bmod Q \\
\chi \neq \chi_{0}}} \chi(A) \\
& =-\frac{(-\log q)^{k}}{\phi(Q)} \sum_{n=1}^{\operatorname{deg} Q-1} n^{k} q^{-\frac{n}{2}} \sum_{\substack{A \in \mathcal{M} \\
\operatorname{deg} A=n}} 1 \\
& =-\frac{(-\log q)^{k}}{q^{\frac{1}{2}}-1} \frac{(\operatorname{deg} Q)^{k}}{|Q|^{\frac{1}{2}}}+O_{k}\left((\log q)^{k} \frac{(\operatorname{deg} Q)^{k-1}}{|Q|^{\frac{1}{2}}}\right) .
\end{aligned}
$$

For the second equality we used Lemma 3.3, and for the last equality we used Lemma 4.1 and the fact that $\phi(Q)=|Q|-1$ (since $Q$ is prime).

\section{Second moments}

Proof of Theorem 2.5 For positive integers $k$ we have that

$$
\begin{aligned}
L^{(k)}\left(\frac{1}{2}, \chi\right) & =(-\log q)^{k} \sum_{n=1}^{\operatorname{deg} Q-1} n^{k} q^{-\frac{n}{2}} \sum_{\substack{A \in \mathcal{M} \\
\operatorname{deg} A=n}} \chi(A) \\
& =(-\log q)^{k} \sum_{\substack{A \in \mathcal{M} \\
\operatorname{deg} A<\operatorname{deg} Q}} \frac{\left(\log _{q}|A|\right)^{k} \chi(A)}{|A|^{\frac{1}{2}}},
\end{aligned}
$$

and so

$$
\begin{aligned}
& \frac{1}{\phi(Q)} \sum_{\substack{\chi \bmod _{\chi \neq} \\
\chi \neq \chi_{0}}}\left|L^{(k)}\left(\frac{1}{2}, \chi\right)\right|^{2} \\
& =\frac{(\log q)^{2 k}}{\phi(Q)} \sum_{\substack{A, B \in \mathcal{M} \\
\operatorname{deg} A, \operatorname{deg} B<\operatorname{deg} Q}} \frac{\left(\log _{q}|A| \log _{q}|B|\right)^{k}}{|A B|^{\frac{1}{2}}} \sum_{\substack{\bmod _{\chi} \\
\chi \neq \chi_{0}}} \chi(A) \bar{\chi}(B) .
\end{aligned}
$$

We now apply Corollary 3.4 to obtain that

$$
\begin{aligned}
& \frac{1}{\phi(Q)} \sum_{\substack{\chi \bmod Q \\
\chi \neq \chi_{0}}}\left|L^{(k)}\left(\frac{1}{2}, \chi\right)\right|^{2} \\
& =(\log q)^{2 k} \sum_{\substack{A \in \mathcal{M} \\
\operatorname{deg} A<\operatorname{deg} Q}} \frac{\left(\log _{q}|A|\right)^{2 k}}{|A|}-\frac{(\log q)^{2 k}}{\phi(Q)} \sum_{\substack{A, B \in \mathcal{M} \\
\operatorname{deg} A, \operatorname{deg} B<\operatorname{deg} Q}} \frac{\left(\log _{q}|A| \log _{q}|B|\right)^{k}}{|A B|^{\frac{1}{2}}} .
\end{aligned}
$$


For the first term on the RHS we have that

$$
\sum_{\substack{A \in \mathcal{M} \\ \operatorname{deg} A<\operatorname{deg} Q}} \frac{\left(\log _{q}|A|\right)^{2 k}}{|A|}=\sum_{n=0}^{\operatorname{deg} Q-1} n^{2 k}=\frac{1}{2 k+1}(\operatorname{deg} Q)^{2 k+1}+O\left((\operatorname{deg} Q)^{2 k}\right),
$$

where the final equality uses Faulhaber's formula. For the second term we have that

$$
\begin{aligned}
& \frac{1}{\phi(Q)} \sum_{\substack{A, B \in \mathcal{M} \\
\operatorname{deg} A, \operatorname{deg} B<\operatorname{deg} Q}} \frac{\left(\log _{q}|A| \log _{q}|B|\right)^{k}}{|A B|^{\frac{1}{2}}}=\frac{1}{\phi(Q)}\left(\sum_{n=0}^{\operatorname{deg} Q-1} n^{k} q^{\frac{n}{2}}\right)^{2} \\
& \leq \frac{1}{\phi(Q)}\left((\operatorname{deg} Q)^{k} \sum_{n=0}^{\operatorname{deg} Q-1} q^{\frac{n}{2}}\right)^{2} \ll \frac{1}{\phi(Q)}\left((\operatorname{deg} Q)^{k}|Q|^{\frac{1}{2}}\right)^{2} \ll(\operatorname{deg} Q)^{2 k} .
\end{aligned}
$$

The result now follows.

\section{Fourth moments: expressing as manageable summations}

Before proceeding to the main part of the proof for the fourth moments, we need to express the fourth moments as more manageable summations.

A generalisation of the following theorem appears in Rosen's book [23, Theorem 9.24 A].

Theorem 6.1 (Functional Equation for $L(s, \chi)$ ) Let $\chi$ be a non-trivial character with modulus $Q \in \mathcal{P}$. If $\chi$ is an odd character, then $L(s, \chi)$ satisfies the functional equation

$$
L(s, \chi)=W(\chi) q^{\frac{\operatorname{deg} Q-1}{2}}\left(q^{-s}\right)^{\operatorname{deg} Q-1} L(1-s, \bar{\chi}),
$$

and if $\chi$ is an even character, then $L(s, \chi)$ satisfies the functional equation

$$
\left(q^{1-s}-1\right) L(s, \chi)=W(\chi) q^{\frac{\operatorname{deg} Q}{2}}\left(q^{-s}-1\right)\left(q^{-s}\right)^{\operatorname{deg} Q-1} L(1-s, \bar{\chi}) ;
$$

where we always have

$$
|W(\chi)|=1 .
$$

Lemma 6.2 Let $\chi$ be an odd character of modulus $Q \in \mathcal{P}$, and let $k$ be a non-negative integer. Then,

$$
\begin{aligned}
& (\log q)^{-2 k}\left|L^{(k)}\left(\frac{1}{2}, \chi\right)\right|^{2} \\
& =\sum_{\substack{A, B \in \mathcal{M} \\
\operatorname{deg} A B<\operatorname{deg} Q}} \frac{\left(f_{k}(\operatorname{deg} A, \operatorname{deg} B, \operatorname{deg} Q)+g_{O, k}(\operatorname{deg} A, \operatorname{deg} B, \operatorname{deg} Q)\right) \chi(A) \bar{\chi}(B)}{|A B|^{\frac{1}{2}}} \\
& +\sum_{\substack{A, B \in \mathcal{M} \\
\operatorname{deg} A B=\operatorname{deg} Q-1}} \frac{h_{O, k}(\operatorname{deg} A, \operatorname{deg} B, \operatorname{deg} Q) \chi(A) \bar{\chi}(B)}{|A B|^{\frac{1}{2}}},
\end{aligned}
$$

where

$$
f_{k}(\operatorname{deg} A, \operatorname{deg} B, \operatorname{deg} Q)=(\operatorname{deg} A)^{k}(\operatorname{deg} B)^{k}+(\operatorname{deg} Q-\operatorname{deg} A)^{k}(\operatorname{deg} Q-\operatorname{deg} B)^{k},
$$




$$
\begin{aligned}
g_{O, k}(\operatorname{deg} A, \operatorname{deg} B, \operatorname{deg} Q)= & (\operatorname{deg} Q-\operatorname{deg} A-1)^{k}(\operatorname{deg} Q-\operatorname{deg} B-1)^{k} \\
& -(\operatorname{deg} Q-\operatorname{deg} A)^{k}(\operatorname{deg} Q-\operatorname{deg} B)^{k}, \\
h_{O, k}(\operatorname{deg} A, \operatorname{deg} B, \operatorname{deg} Q)= & -(\operatorname{deg} Q-\operatorname{deg} A-1)^{k}(\operatorname{deg} Q-\operatorname{deg} B-1)^{k} .
\end{aligned}
$$

Remark 6.3 The " $O$ " in the subscript is to signify that these polynomials apply to the odd character case. It is important to note that $g_{O, k}(\operatorname{deg} A, \operatorname{deg} B, \operatorname{deg} Q)$ has degree $2 k-1$, whereas $f_{k}(\operatorname{deg} A, \operatorname{deg} B, \operatorname{deg} Q)$ has degree $2 k$ (hence, the later ultimately contributes the higher order term); and that all three polynomials are independent of $q$.

Proof The functional equation gives us that

$$
\begin{aligned}
\sum_{n=0}^{\operatorname{deg} Q-1} L_{n}(\chi)\left(q^{-s}\right)^{n} & =W(\chi) q^{\frac{\operatorname{deg} Q-1}{2}}\left(q^{-s}\right)^{\operatorname{deg} Q-1} \sum_{n=0}^{\operatorname{deg} Q-1} L_{n}(\bar{\chi})\left(q^{s-1}\right)^{n} \\
& =W(\chi) q^{-\frac{\operatorname{deg} Q-1}{2}} \sum_{n=0}^{\operatorname{deg} Q-1} L_{n}(\bar{\chi})\left(q^{1-s}\right)^{\operatorname{deg} Q-n-1}
\end{aligned}
$$

Taking the $k^{\text {th }}$ derivative of both sides gives

$$
\begin{aligned}
& (-\log q)^{k} \sum_{n=0}^{\operatorname{deg} Q-1} n^{k} L_{n}(\chi)\left(q^{-s}\right)^{n} \\
& =(-\log q)^{k} W(\chi) q^{-\frac{\operatorname{deg} Q-1}{2}} \sum_{n=0}^{\operatorname{deg} Q-1}(\operatorname{deg} Q-n-1)^{k} L_{n}(\bar{\chi})\left(q^{1-s}\right)^{\operatorname{deg} Q-n-1} .
\end{aligned}
$$

Let us now take the squared modulus of both sides. In order to make our calculations slightly easier, we restrict our attention to the case where $s \in \mathbb{R}$. We obtain

$$
\begin{aligned}
& (\log q)^{2 k} \sum_{n=0}^{2 \operatorname{deg} Q-2}\left(\sum_{\substack{i+j=n \\
0 \leq i, j<\operatorname{deg} Q}} i^{k} j^{k} L_{i}(\chi) L_{j}(\bar{\chi})\right)\left(q^{-s}\right)^{n} \\
& =(\log q)^{2 k} q^{-\operatorname{deg} Q+1} \\
& \sum_{n=0}^{2 \operatorname{deg} Q-2}\left(\sum_{\substack{i+j=n \\
0 \leq i, j<\operatorname{deg} Q}}(\operatorname{deg} Q-i-1)^{k}(\operatorname{deg} Q-j-1)^{k} L_{i}(\chi) L_{j}(\bar{\chi})\right)\left(q^{1-s}\right)^{2 \operatorname{deg} Q-n-2} .
\end{aligned}
$$

Both sides of the above are equal to $\left|L^{(k)}(s, \chi)\right|^{2}$. By the linear independence of powers of $q^{-s}$, we have that $\left|L^{(k)}(s, \chi)\right|^{2}$ is the sum of the terms corresponding to $n=0,1, \ldots, \operatorname{deg} Q-1$ from the LHS and $n=0,1, \ldots, \operatorname{deg} Q-2$ from the RHS. This gives 


$$
\begin{aligned}
& \quad(\log q)^{-2 k}\left|L^{(k)}(s, \chi)\right|^{2} \\
& =\sum_{n=0}^{\operatorname{deg} Q-1}\left(\sum_{\substack{i+j=n \\
0 \leq i, j<\operatorname{deg} Q}} i^{k} j^{k} L_{i}(\chi) L_{j}(\bar{\chi})\right)\left(q^{-s}\right)^{n} \\
& \quad+q^{-\operatorname{deg} Q+1} \sum_{n=0}^{\operatorname{deg} Q-2}\left(\sum_{\substack{i+j=n \\
0 \leq i, j<\operatorname{deg} Q}}(\operatorname{deg} Q-i-1)^{k}(\operatorname{deg} Q-j-1)^{k} L_{i}(\chi) L_{j}(\bar{\chi})\right)\left(q^{1-s}\right)^{2 \operatorname{deg} Q-n-2} .
\end{aligned}
$$

We now substitute $s=\frac{1}{2}$ and simplify the right-hand-side to obtain

$$
\begin{aligned}
& (\log q)^{-2 k}\left|L^{(k)}\left(\frac{1}{2}, \chi\right)\right|^{2} \\
& =\sum_{n=0}^{\operatorname{deg} Q-1}\left(\sum_{\substack{i+j=n \\
0 \leq i, j<\operatorname{deg} Q}} i^{k} j^{k} L_{i}(\chi) L_{j}(\bar{\chi})\right) q^{-\frac{n}{2}} \\
& +\sum_{n=0}^{\operatorname{deg} Q-2}\left(\sum_{\substack{i+j=n \\
0 \leq i, j<\operatorname{deg} Q}}(\operatorname{deg} Q-i-1)^{k}(\operatorname{deg} Q-j-1)^{k} L_{i}(\chi) L_{j}(\bar{\chi})\right) q^{-\frac{n}{2}} \\
& =\sum_{n=0}^{\operatorname{deg} Q-1}\left(\sum_{\substack{i+j=n \\
0 \leq i, j<\operatorname{deg} Q}}\left[i^{k} j^{k}+(\operatorname{deg} Q-i-1)^{k}(\operatorname{deg} Q-j-1)^{k}\right] L_{i}(\chi) L_{j}(\bar{\chi})\right) q^{-\frac{n}{2}} \\
& -\sum_{\substack{i+j=\operatorname{deg} Q-1 \\
0 \leq i, j<\operatorname{deg} Q}}(\operatorname{deg} Q-i-1)^{k}(\operatorname{deg} Q-j-1)^{k} L_{i}(\chi) L_{j}(\bar{\chi}) q^{-\frac{\operatorname{deg} Q-1}{2}} .
\end{aligned}
$$

Finally, we substitute back $L_{n}(\chi)=\sum_{\operatorname{deg} A=\mathcal{M}}^{A \in \mathcal{M}} \chi(A)$ to obtain the required result.

Definition 6.4 For all $s \in \mathbb{C}$ and all non-trivial even characters, $\chi$, of prime modulus we define

$$
\hat{L}(s, \chi):=\left(q^{1-s}-1\right) L(s, \chi)
$$

Lemma 6.5 For all non-trivial even characters, $\chi$, of prime modulus and all non-negative integers $k$ we have that

$$
\begin{aligned}
L^{(k)}\left(\frac{1}{2}, \chi\right) & =\frac{1}{q^{\frac{1}{2}}-1} \hat{L}^{(k)}\left(\frac{1}{2}, \chi\right)+\frac{1}{q^{\frac{1}{2}}-1} \sum_{i=0}^{k-1}(-\log q)^{k-i} p_{k, i}\left(\frac{q^{\frac{1}{2}}}{q^{\frac{1}{2}}-1}\right) \hat{L}^{(i)}\left(\frac{1}{2}, \chi\right) \\
& =\frac{1}{q^{\frac{1}{2}}-1} \sum_{i=0}^{k}(-\log q)^{k-i} p_{k, i}\left(\frac{q^{\frac{1}{2}}}{q^{\frac{1}{2}}-1}\right) \hat{L}^{(i)}\left(\frac{1}{2}, \chi\right),
\end{aligned}
$$

where, for non-negative integers $k, i$ satisfying $i \leq k$, we define the polynomials $p_{k, i}$ by 


$$
\begin{aligned}
& p_{k, k}\left(\frac{q^{\frac{1}{2}}}{q^{\frac{1}{2}}-1}\right)=1, \\
& p_{k, i}\left(\frac{q^{\frac{1}{2}}}{q^{\frac{1}{2}}-1}\right)=-\frac{q^{\frac{1}{2}}}{q^{\frac{1}{2}}-1} \sum_{j=i}^{k-1}\left(\begin{array}{l}
k \\
j
\end{array}\right) p_{j, i}\left(\frac{q^{\frac{1}{2}}}{q^{\frac{1}{2}}-1}\right) \quad \text { for } i<k .
\end{aligned}
$$

Remark 6.6 Because $1 \leq \frac{q^{\frac{1}{2}}}{q^{\frac{1}{2}}-1}<4$ for all prime powers $q$, we can see that the polynomials $p_{k, i}\left(\frac{q^{\frac{1}{2}}}{q^{\frac{1}{2}}-1}\right)$ can be bounded independently of $q$ (but dependent on $k$ and $i$ of course). The factors $(-\log q)^{k-i}$ are of course still dependent on $q$, as well as $k$ and $i$. These two points are important when we later determine how the lower order terms in our main results are dependent on $q$.

Proof We prove this by strong induction on $k$. The base case, $k=0$, is obvious by Definition 6.4. Now, suppose the claim holds for $j=0,1, \ldots, k$. Differentiating, $k+1$ number of times, the Eq. (8) gives

$$
\hat{L}^{(k+1)}(s, \chi)=\left(q^{1-s}-1\right) L^{(k+1)}(s, \chi)+q^{1-s} \sum_{j=0}^{k}\left(\begin{array}{c}
k+1 \\
j
\end{array}\right)(-\log q)^{k+1-j} L^{(j)}(s, \chi) .
$$

Substituting $s=\frac{1}{2}$ and rearranging gives

$$
L^{(k+1)}\left(\frac{1}{2}, \chi\right)=\frac{1}{q^{\frac{1}{2}}-1} \hat{L}^{(k+1)}\left(\frac{1}{2}, \chi\right)-\frac{q^{\frac{1}{2}}}{q^{\frac{1}{2}}-1} \sum_{j=0}^{k}\left(\begin{array}{c}
k+1 \\
j
\end{array}\right)(-\log q)^{k+1-j} L^{(j)}\left(\frac{1}{2}, \chi\right) .
$$

We now apply the inductive hypothesis to obtain

$$
\begin{aligned}
L^{(k+1)}\left(\frac{1}{2}, \chi\right) \\
=\frac{1}{q^{\frac{1}{2}}-1} \hat{L}^{(k+1)}\left(\frac{1}{2}, \chi\right) \\
\quad-\frac{q^{\frac{1}{2}}}{q^{\frac{1}{2}}-1} \sum_{j=0}^{k}\left(\begin{array}{c}
k+1 \\
j
\end{array}\right)(-\log q)^{k+1-j} \frac{1}{q^{\frac{1}{2}}-1} \sum_{i=0}^{j}(-\log q)^{j-i} p_{j, i}\left(\frac{q^{\frac{1}{2}}}{q^{\frac{1}{2}}-1}\right) \hat{L}^{(i)}\left(\frac{1}{2}, \chi\right) \\
=\frac{1}{q^{\frac{1}{2}}-1} \hat{L}^{(k+1)}\left(\frac{1}{2}, \chi\right) \\
\quad+\frac{1}{q^{\frac{1}{2}}-1} \sum_{i=0}^{k}(-\log q)^{k+1-i}\left(-\frac{q^{\frac{1}{2}}}{q^{\frac{1}{2}}-1} \sum_{j=i}^{k}\left(\begin{array}{c}
k+1 \\
j
\end{array}\right) p_{j, i}\left(\frac{q^{\frac{1}{2}}}{q^{\frac{1}{2}}-1}\right)\right) \hat{L}^{(i)}\left(\frac{1}{2}, \chi\right) .
\end{aligned}
$$

The result follows by the definition of the polynomials $p_{k, i}$.

Lemma 6.7 For all non-negative integers $k$, and all non-trivial even characters $\chi$ of modulus $Q \in \mathcal{P}$, we have that 


$$
\begin{aligned}
& \frac{1}{(\log q)^{2 k}\left(q^{\frac{1}{2}}-1\right)^{2}}\left|\hat{L}^{(k)}\left(\frac{1}{2}, \chi\right)\right|^{2} \\
& =\sum_{\substack{A, B \in \mathcal{M} \\
\operatorname{deg} A B<\operatorname{deg} Q}} \frac{\left(f_{k}(\operatorname{deg} A, \operatorname{deg} B, \operatorname{deg} Q)+g_{E, k}(\operatorname{deg} A, \operatorname{deg} B, \operatorname{deg} Q)\right) \chi(A) \bar{\chi}(B)}{|A B|^{\frac{1}{2}}} \\
& +\sum_{\operatorname{deg} Q-2 \leq n \leq \operatorname{deg} Q} \sum_{\substack{A, B \in \mathcal{M} \\
\operatorname{deg} A B=n}} \frac{h_{E, k, n}(\operatorname{deg} A, \operatorname{deg} B, \operatorname{deg} Q) \chi(A) \bar{\chi}(B)}{|A B|^{\frac{1}{2}}},
\end{aligned}
$$

where

$$
f_{k}(\operatorname{deg} A, \operatorname{deg} B, \operatorname{deg} Q)=(\operatorname{deg} A)^{k}(\operatorname{deg} B)^{k}+(\operatorname{deg} Q-\operatorname{deg} A)^{k}(\operatorname{deg} Q-\operatorname{deg} B)^{k},
$$

and $g_{E, k}(\operatorname{deg} A, \operatorname{deg} B, \operatorname{deg} Q), h_{E, k, n}(\operatorname{deg} A, \operatorname{deg} B, \operatorname{deg} Q)$ are polynomials of degrees $2 k-1$ and $2 k$, respectively, whose coefficients can be bounded independently of $q$.

Proof Let us define $L_{-1}(\chi):=0$, and recall from Lemma 3.6 that $L_{\operatorname{deg} Q}(\chi)=0$. We can now define, for $n=0,1, \ldots, \operatorname{deg} Q$,

$$
M_{n}(\chi):=L_{n}(\chi)-q L_{n-1}(\chi) .
$$

Then, the functional equation for even characters can be written as

$$
-\sum_{n=0}^{\operatorname{deg} Q} M_{n}(\chi)\left(q^{-s}\right)^{n}=W(\chi) q^{-\frac{\operatorname{deg} Q}{2}} \sum_{n=0}^{\operatorname{deg} Q} M_{n}(\bar{\chi})\left(q^{1-s}\right)^{\operatorname{deg} Q-n} .
$$

Note that both sides of (9) are equal to $\hat{L}(s, \chi)$. We proceed similarly to the odd character case. First we differentiate, $k$ number of times, the Eq. (9); and then we take the modulus squared of both sides. Again, in order to make our calculations slightly easier, we restrict our attention to the case where $s \in \mathbb{R}$. We obtain

$$
\begin{aligned}
& (\log q)^{2 k} \sum_{n=0}^{2 \operatorname{deg} Q}\left(\sum_{\substack{i+j=n \\
0 \leq i, j \leq \operatorname{deg} Q}} i^{k} j^{k} M_{i}(\chi) M_{j}(\bar{\chi})\right)\left(q^{-s}\right)^{n} \\
& =(\log q)^{2 k} q^{-\operatorname{deg} Q} \sum_{n=0}^{2 \operatorname{deg} Q}\left(\sum_{\substack{i+j=n \\
0 \leq i, j \leq \operatorname{deg} Q}}(\operatorname{deg} Q-i)^{k}(\operatorname{deg} Q-j)^{k} M_{i}(\chi) M_{j}(\bar{\chi})\right)\left(q^{1-s}\right)^{2 \operatorname{deg} Q-n} .
\end{aligned}
$$

Now we take the terms corresponding to $n=0,1, \ldots, \operatorname{deg} Q$ from the LHS and $n=$ $0,1, \ldots, \operatorname{deg} Q-1$ from the RHS to obtain

$$
\begin{aligned}
\hat{L}^{(k)}(s, \chi)= & (\log q)^{2 k} \sum_{n=0}^{\operatorname{deg} Q}\left(\sum_{\substack{i+j=n \\
0 \leq i, j \leq \operatorname{deg} Q}} i^{k} j^{k} M_{i}(\chi) M_{j}(\bar{\chi})\right)\left(q^{-s}\right)^{n} \\
& +(\log q)^{2 k} q^{-\operatorname{deg} Q} \sum_{n=0}^{\operatorname{deg} Q-1}\left(\sum_{\substack{i+j=n \\
0 \leq i, j \leq \operatorname{deg} Q}}(\operatorname{deg} Q-i)^{k}(\operatorname{deg} Q-j)^{k} M_{i}(\chi) M_{j}(\bar{\chi})\right)\left(q^{1-s}\right)^{2 \operatorname{deg} Q-n} .
\end{aligned}
$$


Substituting $s=\frac{1}{2}$ and simplifying the RHS gives

$$
\begin{aligned}
\hat{L}^{(k)}\left(\frac{1}{2}, \chi\right)= & (\log q)^{2 k} \sum_{n=0}^{\operatorname{deg} Q-1}\left(\sum_{\substack{i+j=n \\
0 \leq i, j \leq \operatorname{deg} Q}}\left(i^{k} j^{k}+(\operatorname{deg} Q-i)^{k}(\operatorname{deg} Q-j)^{k}\right) M_{i}(\chi) M_{j}(\bar{\chi})\right) q^{-\frac{n}{2}} \\
& +(\log q)^{2 k} \sum_{\substack{i+j=\operatorname{deg} Q \\
0 \leq i, j \leq \operatorname{deg} Q}} i^{k} j^{k} M_{i}(\chi) M_{j}(\bar{\chi}) q^{-\frac{\operatorname{deg} Q}{2}} .
\end{aligned}
$$

Now, we want factors such as $L_{n}(\chi)$ in our expression, as opposed to factors like $M_{n}(\chi)$. To this end, suppose $p(i, j)$ is a finite polynomial. Then,

$$
\begin{aligned}
& \sum_{n=0}^{\operatorname{deg} Q-1}\left(\sum_{\substack{i+j=n \\
0 \leq i, j \leq \operatorname{deg} Q}} p(i, j) M_{i}(\chi) M_{j}(\bar{\chi})\right) q^{-\frac{n}{2}} \\
& =\sum_{n=0}^{\operatorname{deg} Q-1}\left(\sum_{\substack{i+j=n \\
0 \leq i, j \leq \operatorname{deg} Q}} p(i, j)\left(L_{i}(\chi)-q L_{i-1}(\chi)\right)\left(L_{j}(\bar{\chi})-q L_{j-1}(\bar{\chi})\right)\right) q^{-\frac{n}{2}} \\
& =\sum_{n=0}^{\operatorname{deg} Q-1}\left(\sum_{\substack{i+j=n \\
0 \leq i, j \leq \operatorname{deg} Q}} p(i, j) L_{i}(\chi) L_{j}(\bar{\chi})\right) q^{-\frac{n}{2}} \\
& +\sum_{n=0}^{\operatorname{deg} Q-3}\left(\sum_{\substack{i+j=n \\
0 \leq i, j \leq \operatorname{deg} Q}} p(i+1, j+1) L_{i}(\chi) L_{j}(\bar{\chi})\right) q^{-\frac{n-2}{2}} \\
& -\sum_{n=0}^{\operatorname{deg} Q-2}\left(\sum_{\substack{i+j=n \\
0 \leq i, j \leq \operatorname{deg} Q}} p(i, j+1) L_{i}(\chi) L_{j}(\bar{\chi})\right) q^{-\frac{n-1}{2}} \\
& -\sum_{n=0}^{\operatorname{deg} Q-2}\left(\sum_{\substack{i+j=n \\
0 \leq i, j \leq \operatorname{deg} Q}} p(i+1, j) L_{i}(\chi) L_{j}(\bar{\chi})\right) q^{-\frac{n-1}{2}} .
\end{aligned}
$$

Grouping the terms together gives

$$
\begin{aligned}
& \sum_{n=0}^{\operatorname{deg} Q-1}\left(\sum_{\substack{i+j=n \\
0 \leq i, j \leq \operatorname{deg} Q}} p(i, j) M_{i}(\chi) M_{j}(\bar{\chi})\right) q^{-\frac{n}{2}} \\
& =\sum_{n=0}^{\operatorname{deg} Q-1}\left(\sum _ { \substack { i + j = n \\
0 \leq i , j \leq \operatorname { d e g } Q } } \left[q p(i+1, j+1)-q^{\frac{1}{2}} p(i, j+1)\right.\right. \\
& \\
& \left.\left.\quad-q^{\frac{1}{2}} p(i+1, j)+p(i, j)\right] L_{i}(\chi) L_{j}(\bar{\chi})\right) q^{-\frac{n}{2}}
\end{aligned}
$$




$$
\begin{aligned}
& -\sum_{\substack{i+j=\operatorname{deg} Q-2 \\
0 \leq i, j \leq \operatorname{deg} Q}} q p(i+1, j+1) L_{i}(\chi) L_{j}(\bar{\chi}) q^{-\frac{\operatorname{deg} Q-2}{2}} \\
& +\sum_{\substack{i+j=\operatorname{deg} Q-1 \\
0 \leq i, j \leq \operatorname{deg} Q}}\left(q^{\frac{1}{2}} p(i, j+1)+q^{\frac{1}{2}} p(i+1, j)-q p(i+1, j+1)\right) L_{i}(\chi) L_{j}(\bar{\chi}) q^{\frac{\operatorname{deg} Q-1}{2}} .
\end{aligned}
$$

In the case where

$$
p(i, j)=i^{k} j^{k}+(\operatorname{deg} Q-i)^{k}(\operatorname{deg} Q-j)^{k}
$$

we have that

$$
\begin{aligned}
& q p(i+1, j+1)-q^{\frac{1}{2}} p(i, j+1)-q^{\frac{1}{2}} p(i+1, j)+p(i, j) \\
& =\left(q^{\frac{1}{2}}-1\right)^{2}\left(f_{k}(i, j, \operatorname{deg} Q)+g_{E, k}(i, j, \operatorname{deg} Q)\right),
\end{aligned}
$$

where $g_{E, k}(i, j, \operatorname{deg} Q)$ is a polynomial of degree $2 k-1$ whose coefficients can be bounded independently of $q$.

We can now see that (10) becomes

$$
\begin{aligned}
& \frac{1}{(\log q)^{2 k}\left(q^{\frac{1}{2}}-1\right)^{2}} \hat{L}^{(k)}\left(\frac{1}{2}, \chi\right) \\
& =\sum_{n=0}^{\operatorname{deg} Q-1}\left(\sum_{\substack{i+j=n \\
0 \leq i, j \leq \operatorname{deg} Q}}\left(f_{k}(i, j, \operatorname{deg} Q)+g_{E, k}(i, j, \operatorname{deg} Q)\right) L_{i}(\chi) L_{j}(\bar{\chi})\right) q^{-\frac{n}{2}} \\
& +\sum_{n=\operatorname{deg} Q-2}^{\operatorname{deg} Q}\left(\sum_{\substack{i+j=n \\
0 \leq i, j \leq \operatorname{deg} Q}} h_{E, k, n}(i, j, \operatorname{deg} Q) L_{i}(\chi) L_{j}(\bar{\chi})\right) q^{-\frac{n}{2}},
\end{aligned}
$$

where $h_{E, k, n}(i, j, \operatorname{deg} Q)$ is a polynomial of degree $k$ whose coefficients can be bounded independently of $q$. Finally, we substitute back $L_{n}(\chi)=\sum_{\operatorname{deg} A=n}^{A \in \mathcal{M}} \underset{(A)}{ } \chi($ to obtain the required result.

\section{Fourth moments: handling the summations}

We now demonstrate some techniques for handling the summations that we obtained in Sect. 6.

Lemma 7.1 Let $Q \in \mathcal{P}$, and let $p_{1}(\operatorname{deg} A, \operatorname{deg} B, \operatorname{deg} Q)$ and $p_{2}(\operatorname{deg} A, \operatorname{deg} B, \operatorname{deg} Q)$ be finite polynomials (which, for presentational purposes, we will write as $p_{1}$ and $p_{2}$, except when we need to use variables other than $\operatorname{deg} A, \operatorname{deg} B, \operatorname{deg} Q)$. Then, 


$$
\begin{aligned}
& \frac{1}{\phi(Q)} \sum_{\substack{\chi \bmod Q \\
\chi \neq \chi_{0}}}\left(\sum_{\substack{A, B \in \mathcal{M} \\
\operatorname{deg} A B<\operatorname{deg} Q}} \frac{p_{1} \chi(A) \bar{\chi}(B)}{|A B|^{\frac{1}{2}}}\right)\left(\sum_{\substack{C, D \in \mathcal{M} \\
\operatorname{deg} C D<\operatorname{deg} Q}} \frac{p_{2} \chi(C) \bar{\chi}(D)}{|C D|^{\frac{1}{2}}}\right) \\
& =\sum_{\substack{A, B, C, D \in \mathcal{M} \\
\operatorname{deg} A B<\operatorname{deg} Q \\
\operatorname{deg} C D<\operatorname{deg} Q \\
A C=B D}} \frac{p_{1} p_{2}}{|A B C D|^{\frac{1}{2}}}+\sum_{\begin{array}{c}
A, B, C, D \in \mathcal{M} \\
\operatorname{deg} A B<\operatorname{deg} Q \\
\operatorname{deg} C D<\operatorname{deg} Q \\
A C \equiv B D(\operatorname{mog} Q) \\
A C \neq B D
\end{array}} \frac{p_{1} p_{2}}{|A B C D|^{\frac{1}{2}}}-\frac{1}{\phi(Q)} \sum_{\begin{array}{c}
A, B, C, D \in \mathcal{M} \\
\operatorname{deg} A B<\operatorname{deg} Q \\
\operatorname{deg} C D<\operatorname{deg} Q
\end{array}} \frac{p_{1} p_{2}}{|A B C D|^{\frac{1}{2}}} .
\end{aligned}
$$

Proof This follows by expanding the brackets and applying Corollary 3.4 .

Lemma 7.2 Let $p(\operatorname{deg} A, \operatorname{deg} B, \operatorname{deg} C, \operatorname{deg} D, \operatorname{deg} Q)$ be a finite homogeneous polynomial of degree $d$. Then,

$$
\begin{aligned}
& \quad \sum_{\substack{A, B, C, D \in \mathcal{M} \\
\operatorname{deg} A B<\operatorname{deg} Q \\
\operatorname{deg} C D<\operatorname{deg} Q \\
A C=B D}} \frac{p(\operatorname{deg} A, \operatorname{deg} B, \operatorname{deg} C, \operatorname{deg} D, \operatorname{deg} Q)}{|A B C D|^{\frac{1}{2}}} \\
& =\left(1-q^{-1}\right)(\operatorname{deg} Q)^{d+4} \\
& \quad \times \int_{\substack{a_{1}, a_{2}, a_{3}, a_{4} \geq 0 \\
2 a_{1}+a_{3}+a_{4}<1 \\
2 a_{2}+a_{3}+a_{4}<1}} p\left(a_{1}+a_{3}, a_{1}+a_{4}, a_{2}+a_{4}, a_{2}+a_{3}, 1\right) \mathrm{d} a_{1} \mathrm{~d} a_{2} \mathrm{~d} a_{3} \mathrm{~d} a_{4}+O_{p}\left((\operatorname{deg} Q)^{d+3}\right) .
\end{aligned}
$$

Remark 7.3 The subscript $p$ in $O_{p}$ should be interpreted as saying that the implied constant is dependent on the coefficients of $p$.

Proof Consider the function $f$ defined by

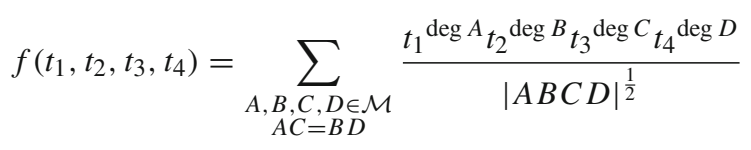

with domain $\left|t_{i}\right|<\frac{1}{2} q^{-\frac{1}{2}}$. Note that $A C=B D$ if and only if there exist $G, H, R, S \in \mathcal{M}$ satisfying $(R, S)=1$ and $A=G R, B=G S, C=H S, D=H R$. Hence,

$$
\begin{aligned}
& f\left(t_{1}, t_{2}, t_{3}, t_{4}\right) \\
& =\sum_{\substack{G, H, R, S \in \mathcal{M} \\
(R, S)=1}} \frac{t_{1}{ }^{\operatorname{deg} G R} t_{2} \operatorname{deg} G S_{t_{3}} \operatorname{deg} H S_{t_{4}} \operatorname{deg} H R}{|G H R S|} \\
& =\sum_{G, H, R, S \in \mathcal{M}} \frac{t_{1}{ }^{\operatorname{deg} G R} t_{2} \operatorname{deg} G S_{t_{3}} \operatorname{deg} H S_{t_{4}} \operatorname{deg} H R}{|G H R S|}
\end{aligned}
$$

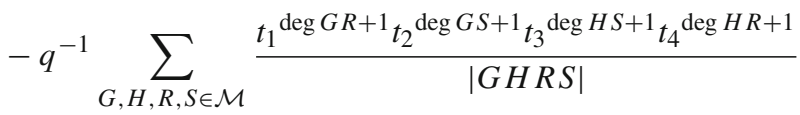

$$
\begin{aligned}
& =\sum_{a_{1}, a_{2}, a_{3}, a_{4} \geq 0} t_{1}{ }^{a_{1}+a_{3}} t_{2}{ }^{a_{1}+a_{4}} t_{3}{ }^{a_{2}+a_{4}} t_{4}{ }^{a_{2}+a_{3}} \\
& -q^{-1} \sum_{a_{1}, a_{2}, a_{3}, a_{4} \geq 0} t_{1}{ }^{a_{1}+a_{3}+1} t_{2}{ }^{a_{1}+a_{4}+1} t_{3}{ }^{a_{2}+a_{4}+1} t_{4}{ }^{a_{2}+a_{3}+1},
\end{aligned}
$$


where the second equality follows by similar means as in the proof of Lemma 3.7.

Now, for $i=1,2,3,4$ we define the operator $\Omega_{i}:=t_{i} \frac{\mathrm{d}}{\mathrm{d} t_{i}}$. For non-negative integers $k_{1}, k_{2}, k_{3}, k_{4}$ we can apply the operator $\Omega_{1}{ }^{k_{1}} \Omega_{2}{ }^{k_{2}} \Omega_{3}{ }^{k_{3}} \Omega_{4}{ }^{k_{4}}$ to (11) and (12) to obtain

$$
\begin{aligned}
& \sum_{\substack{A, B, C, D \in \mathcal{M} \\
A C=B D}} \frac{(\operatorname{deg} A)^{k_{1}}(\operatorname{deg} B)^{k_{2}}(\operatorname{deg} C)^{k_{3}}(\operatorname{deg} D)^{k_{4}}}{|A B C D|^{\frac{1}{2}}} t_{1} \operatorname{deg} A t_{2} \operatorname{deg} B t_{3} \operatorname{deg} C_{t_{4}} \operatorname{deg} D \\
& =\sum_{a_{1}, a_{2}, a_{3}, a_{4} \geq 0}\left(a_{1}+a_{3}\right)^{k_{1}}\left(a_{1}+a_{4}\right)^{k_{2}}\left(a_{2}+a_{4}\right)^{k_{3}}\left(a_{2}+a_{3}\right)^{k_{4}} t_{1}{ }^{a_{1}+a_{3}} t_{2}{ }^{a_{1}+a_{4}} t_{3}{ }^{a_{2}+a_{4}} t_{4}{ }^{a_{2}+a_{3}} \\
& -q^{-1} \sum_{a_{1}, a_{2}, a_{3}, a_{4} \geq 0}\left(a_{1}+a_{3}+1\right)^{k_{1}}\left(a_{1}+a_{4}+1\right)^{k_{2}}\left(a_{2}+a_{4}+1\right)^{k_{3}}\left(a_{2}+a_{3}+1\right)^{k_{4}} \\
& \times t_{1}{ }^{a_{1}+a_{3}+1} t_{2}{ }^{a_{1}+a_{4}+1} t_{3}{ }^{a_{2}+a_{4}+1} t_{4}{ }^{a_{2}+a_{3}+1} \\
& =\left(1-q^{-1}\right) \sum_{a_{1}, a_{2}, a_{3}, a_{4} \geq 0}\left(a_{1}+a_{3}\right)^{k_{1}}\left(a_{1}+a_{4}\right)^{k_{2}}\left(a_{2}+a_{4}\right)^{k_{3}}\left(a_{2}+a_{3}\right)^{k_{4}} \\
& \times t_{1}{ }^{a_{1}+a_{3}} t_{2}{ }^{a_{1}+a_{4}} t_{3}{ }^{a_{2}+a_{4}} t_{4}{ }^{a_{2}+a_{3}} \\
& +q^{-1} \sum_{\substack{\left(a_{1}, a_{2}\right)=(0,0),(0,1),(1,0) \\
a_{3}, a_{4} \geq 0}}\left(a_{1}+a_{3}\right)^{k_{1}}\left(a_{1}+a_{4}\right)^{k_{2}}\left(a_{2}+a_{4}\right)^{k_{3}}\left(a_{2}+a_{3}\right)^{k_{4}} \\
& \times t_{1}{ }^{a_{1}+a_{3}} t_{2}{ }^{a_{1}+a_{4}} t_{3}{ }^{a_{2}+a_{4}} t_{4}{ }^{a_{2}+a_{3}} .
\end{aligned}
$$

From this we can deduce that if $p(\operatorname{deg} A, \operatorname{deg} B, \operatorname{deg} C, \operatorname{deg} D, \operatorname{deg} Q)$ is a finite homogeneous polynomial of degree $d$, then

$$
\begin{aligned}
& \sum_{\substack{A, B, C, D \in \mathcal{M} \\
A C=B D}} \frac{p(\operatorname{deg} A, \operatorname{deg} B, \operatorname{deg} C, \operatorname{deg} D, \operatorname{deg} Q)}{|A B C D|^{\frac{1}{2}}} t_{1} \operatorname{deg} A_{t_{2}} \operatorname{deg} B t_{3} \operatorname{deg} C_{t_{4}} \operatorname{deg} D \\
& =\left(1-q^{-1}\right) \sum_{\substack{a_{1}, a_{2}, a_{3}, a_{4} \geq 0\\
}} p\left(a_{1}+a_{3}, a_{1}+a_{4}, a_{2}+a_{4}, a_{2}+a_{3}, \operatorname{deg} Q\right) t_{1}{ }^{a_{1}+a_{3}} t_{2}{ }^{a_{1}+a_{4}} t_{3}{ }^{a_{2}+a_{4}} t_{4}{ }^{a_{2}+a_{3}} \\
& +q^{-1} \sum_{\substack{\left(a_{1}, a_{2}\right)=(0,0),(0,1),(1,0) \\
a_{3}, a_{4} \geq 0}} p\left(a_{1}+a_{3}, a_{1}+a_{4}, a_{2}+a_{4}, a_{2}+a_{3}, \operatorname{deg} Q\right) t_{1}{ }^{a_{1}+a_{3}} t_{2}{ }^{a_{1}+a_{4}} t_{3}{ }^{a_{2}+a_{4}} t_{4}{ }^{a_{2}+a_{3}} .
\end{aligned}
$$

Now, we can extract and sum the coefficients of $t_{1}{ }^{i_{1}} t_{2}{ }^{i_{2}} t_{3}{ }^{i_{3}} t_{4}{ }^{i_{4}}$ for which $i_{1}+i_{2}<\operatorname{deg} Q$ and $i_{3}+i_{4}<\operatorname{deg} Q$ to obtain

$$
\begin{aligned}
& \sum_{\substack{A, B, C, D \in \mathcal{M} \\
\operatorname{deg} A B<\operatorname{deg} Q \\
\operatorname{deg} C D<\operatorname{deg} Q \\
A C=B D}} \frac{p(\operatorname{deg} A, \operatorname{deg} B, \operatorname{deg} C, \operatorname{deg} D, \operatorname{deg} Q)}{|A B C D|^{\frac{1}{2}}} \\
& =\left(1-q^{-1}\right) \sum_{\substack{a_{1}, a_{2}, a_{3}, a_{4} \geq 0 \\
2 a_{1}+a_{3}+a_{4}<\operatorname{deg} Q \\
2 a_{2}+a_{3}+a_{4}<\operatorname{deg} Q}} p\left(a_{1}+a_{3}, a_{1}+a_{4}, a_{2}+a_{4}, a_{2}+a_{3}, \operatorname{deg} Q\right) \\
& +q^{-1 \quad} \\
& \sum_{\substack{\left(a_{1}, a_{2}\right)=(0,0),(0,1),(1,0) \\
a_{3}, a_{4} \geq 0 \\
2 a_{1}+a_{3}+a_{4}<\operatorname{deg} Q \\
2 a_{2}+a_{3}+a_{4}<\operatorname{deg} Q}} p\left(a_{1}+a_{3}, a_{1}+a_{4}, a_{2}+a_{4}, a_{2}+a_{3}, \operatorname{deg} Q\right) \\
& =\left(1-q^{-1}\right) \int_{\substack{a_{1}, a_{2}, a_{3}, a_{4} \geq 0 \\
2 a_{1}+a_{3}+a_{4}<\operatorname{deg} Q \\
2 a_{2}+a_{3}+a_{4}<\operatorname{deg} Q}} p\left(a_{1}+a_{3}, a_{1}+a_{4}, a_{2}+a_{4}, a_{2}+a_{3}, \operatorname{deg} Q\right) \mathrm{d} a_{1} \mathrm{~d} a_{2} \mathrm{~d} a_{3} \mathrm{~d} a_{4}
\end{aligned}
$$




$$
\begin{aligned}
& +O_{p}\left((\operatorname{deg} Q)^{d+3}\right)+O_{p}\left((\operatorname{deg} Q)^{d+2}\right) \\
= & \left(1-q^{-1}\right)(\operatorname{deg} Q)^{d+4} \int_{\substack{a_{1}, a_{2}, a_{3}, a_{4} \geq 0 \\
2 a_{1}+a_{3}+a_{4}<1 \\
2 a_{2}+a_{3}+a_{4}<1}} p\left(a_{1}+a_{3}, a_{1}+a_{4}, a_{2}+a_{4}, a_{2}+a_{3}, 1\right) \mathrm{d} a_{1} \mathrm{~d} a_{2} \mathrm{~d} a_{3} \mathrm{~d} a_{4} \\
& +O_{p}\left((\operatorname{deg} Q)^{d+3}\right) .
\end{aligned}
$$

Lemma 7.4 Let $p(\operatorname{deg} A, \operatorname{deg} B, \operatorname{deg} C, \operatorname{deg} D, \operatorname{deg} Q)$ be a finite polynomial of degree $d$. Then,

$$
\begin{aligned}
& \sum_{A, B, C, D \in \mathcal{M}} \frac{p(\operatorname{deg} A, \operatorname{deg} B, \operatorname{deg} C, \operatorname{deg} D, \operatorname{deg} Q)}{|A B C D|^{\frac{1}{2}}} \ll_{p}(\operatorname{deg} Q)^{d+3} . \\
& \operatorname{deg} A B<\operatorname{deg} Q \\
& \operatorname{deg} C D<\operatorname{deg} Q \\
& A C \equiv B D(\bmod Q) \\
& A C \neq B D
\end{aligned}
$$

Proof Because deg $A B, \operatorname{deg} C D<\operatorname{deg} Q$, we have that

$$
p(\operatorname{deg} A, \operatorname{deg} B, \operatorname{deg} C, \operatorname{deg} D, \operatorname{deg} Q) \ll_{p}(\operatorname{deg} Q)^{d} .
$$

Hence,

$$
\begin{gathered}
\sum_{\begin{array}{c}
A, B, C, D \in \mathcal{M} \\
\operatorname{deg} A B<\operatorname{deg} Q \\
\operatorname{deg} C D<\operatorname{deg} Q \\
A C \equiv B D(\bmod Q) \\
A C \neq B D
\end{array}} \frac{p(\operatorname{deg} A, \operatorname{deg} B, \operatorname{deg} C, \operatorname{deg} D, \operatorname{deg} Q)}{|A B C D|^{\frac{1}{2}}} \\
\ll_{p}(\operatorname{deg} Q)^{d} \sum_{\begin{array}{c}
A, B, C, D \in \mathcal{M} \\
\operatorname{deg} A B<\operatorname{deg} Q \\
\operatorname{deg} C D<\operatorname{deg} Q \\
A C \equiv B D(\bmod Q) \\
A C \neq B D
\end{array}} \frac{1}{|A B C D|^{\frac{1}{2}}}=(\operatorname{deg} Q)^{d} \sum_{0 \leq z_{1}, z_{2}<\operatorname{deg} Q} q^{-\frac{z_{1}+z_{2}}{2}} \sum_{\begin{array}{c}
A, B, C, D \in \mathcal{M} \\
\operatorname{deg} A B=z_{1} \\
\operatorname{deg} C D=z_{2} \\
A C \equiv B D(\bmod Q) \\
A C \neq B D
\end{array}} 1 .
\end{gathered}
$$

Now, Lemma 7.9 from [3] tells us that for non-negative integers $z_{1}, z_{2}$ we have

$$
\sum_{\substack{A, B, C, D \in \mathcal{M} \\ \operatorname{deg} A B=z_{1} \\ \operatorname{deg} C D=z_{2} \\ A C \equiv B D(\bmod Q) \\ A C \neq B D}} 1 \begin{cases}\ll_{\epsilon} \frac{1}{|Q|}\left(q^{z_{1}} q^{z_{2}}\right)^{1+\epsilon} & \text { if } z_{1}+z_{2} \leq \frac{19}{10} \operatorname{deg} Q \\ \ll \frac{1}{\phi(Q)} q^{z_{1}} q^{z_{2}}\left(z_{1}+z_{2}\right)^{3} & \text { if } z_{1}+z_{2}>\frac{19}{10} \operatorname{deg} Q .\end{cases}
$$

Hence, for $\epsilon<\frac{1}{38}$ we have

$$
\begin{aligned}
& \sum_{0 \leq z_{1}, z_{2}<\operatorname{deg} Q} q^{-\frac{z_{1}+z_{2}}{2}} \sum_{\begin{array}{c}
A, B, C, D \in \mathcal{M} \\
\operatorname{deg} A B=z_{1} \\
\operatorname{deg} C D=z_{2} \\
C \equiv B D(\bmod Q) \\
A C \neq B D
\end{array}} 1 \\
& \ll \frac{1}{|Q|} \sum_{\substack{A \leq z_{1}, z_{2}<\operatorname{deg} Q \\
z_{1}+z_{2} \leq \frac{19}{10} \operatorname{deg} Q}}\left(q^{\frac{1}{2}+\epsilon}\right)^{z_{1}+z_{2}}+\frac{1}{\phi(Q)} \sum_{\substack{0 \leq z_{1}, z_{2}<\operatorname{deg} Q \\
z_{1}+z_{2}>\frac{19}{10} \operatorname{deg} Q}} q^{\frac{z_{1}+z_{2}}{2}\left(z_{1}+z_{2}\right)^{3}}
\end{aligned}
$$




$$
\ll \frac{|Q|}{\phi(Q)}(\operatorname{deg} Q)^{3} \ll(\operatorname{deg} Q)^{3} .
$$

The result follows by applying this to (13).

Remark 7.5 In her paper, Tamam [25, Lemma 8.5] states a similar result as (14) above. However, in her proof she claims that $d(N) \ll \operatorname{deg} N$, which is not the case. Addressing this is non-trivial and was done in [3], as stated above.

Lemma 7.6 Let $p(\operatorname{deg} A, \operatorname{deg} B, \operatorname{deg} C, \operatorname{deg} D, \operatorname{deg} Q)$ be a finite polynomial of degree $d$. Then,

$$
\frac{1}{\phi(Q)} \sum_{\substack{A, B, C, D \in \mathcal{M} \\ \operatorname{deg} A B<\operatorname{deg} Q \\ \operatorname{deg} C D<\operatorname{deg} Q}} \frac{p(\operatorname{deg} A, \operatorname{deg} B, \operatorname{deg} C, \operatorname{deg} D, \operatorname{deg} Q)}{|A B C D|^{\frac{1}{2}}} \ll_{p}(\operatorname{deg} Q)^{d+2} .
$$

Proof Because $\operatorname{deg} A B, \operatorname{deg} C D<\operatorname{deg} Q$, we have that

$$
p(\operatorname{deg} A, \operatorname{deg} B, \operatorname{deg} C, \operatorname{deg} D, \operatorname{deg} Q) \ll_{p}(\operatorname{deg} Q)^{d} .
$$

Hence,

$$
\begin{aligned}
& \frac{1}{\phi(Q)} \sum_{\substack{A, B, C, D \in \mathcal{M} \\
\operatorname{deg} A B<\operatorname{deg} Q \\
\operatorname{deg} C D<\operatorname{deg} Q}} \frac{p(\operatorname{deg} A, \operatorname{deg} B, \operatorname{deg} C, \operatorname{deg} D, \operatorname{deg} Q)}{|A B C D|^{\frac{1}{2}}} \\
& \ll p \frac{(\operatorname{deg} Q)^{d}}{\phi(Q)}\left(\sum_{\substack{A, B \in \mathcal{M} \\
\operatorname{deg} A B<\operatorname{deg} Q}}^{|A B|^{\frac{1}{2}}}\right)\left(\sum_{\substack{C, D \in \mathcal{M} \\
\operatorname{deg} C D<\operatorname{deg} Q}} \frac{1}{|C D|^{\frac{1}{2}}}\right) \\
& =\frac{(\operatorname{deg} Q)^{d}}{\phi(Q)}\left(\sum_{\substack{n, m \geq 0 \\
n+m<\operatorname{deg} Q}} q^{\frac{m+n}{2}}\right)^{2} \ll(\operatorname{deg} Q)^{d+2} .
\end{aligned}
$$

From Lemmas 7.1 to 7.6 we can deduce the following:

Lemma 7.7 Let $Q \in \mathcal{P}$, and let $p_{1}(\operatorname{deg} A, \operatorname{deg} B, \operatorname{deg} Q)$ and $p_{2}(\operatorname{deg} C, \operatorname{deg} D, \operatorname{deg} Q)$ be finite homogeneous polynomials of degree $d_{1}$ and $d_{2}$, respectively. Then,

$$
\begin{aligned}
& \frac{1}{\phi(Q)} \sum_{\substack{\chi \bmod _{\chi \neq \chi_{0}} \\
\chi}}\left(\sum_{\substack{A, B \in \mathcal{M} \\
\operatorname{deg} A B<\operatorname{deg} Q}} \frac{p_{1} \chi(A) \bar{\chi}(B)}{|A B|^{\frac{1}{2}}}\right)\left(\sum_{\substack{C, D \in \mathcal{M} \\
\operatorname{deg} C D<\operatorname{deg} Q}} \frac{p_{2} \chi(C) \bar{\chi}(D)}{|C D|^{\frac{1}{2}}}\right) \\
& =\left(1-q^{-1}\right)(\operatorname{deg} Q)^{d_{1}+d_{2}+4} \\
& \quad \times \int_{\substack{a_{1}, a_{2}, a_{3}, a_{4} \geq 0 \\
2 a_{1}+a_{3}+a_{4}<1 \\
2 a_{2}+a_{3}+a_{4}<1}} p_{1}\left(a_{1}+a_{3}, a_{1}+a_{4}, 1\right) p_{2}\left(a_{2}+a_{4}, a_{2}+a_{3}, 1\right) \mathrm{d} a_{1} \mathrm{~d} a_{2} \mathrm{~d} a_{3} \mathrm{~d} a_{4} \\
& +O_{p_{1}, p_{2}}\left((\operatorname{deg} Q)^{d_{1}+d_{2}+3}\right) .
\end{aligned}
$$


Similarly, the following can be proved:

Lemma 7.8 Let $Q \in \mathcal{P}$, and let $p_{1}(\operatorname{deg} A, \operatorname{deg} B, \operatorname{deg} Q)$ and $p_{2}(\operatorname{deg} C, \operatorname{deg} D, \operatorname{deg} Q)$ be finite homogeneous polynomials of degree $d_{1}$ and $d_{2}$, respectively. Then,

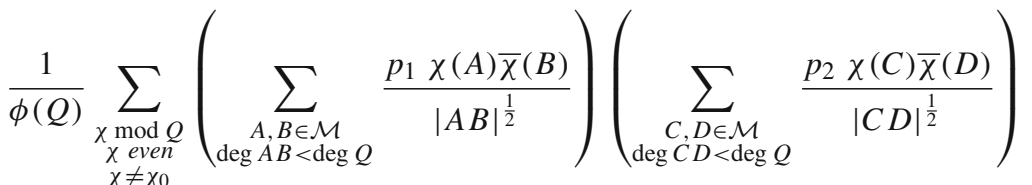

$$
\begin{aligned}
& =q^{-1}(\operatorname{deg} Q)^{d_{1}+d_{2}+4} \\
& \times \int_{\substack{a_{1}, a_{2}, a_{3}, a_{4} \geq 0 \\
2 a_{1}+a_{3}+a_{4}<1 \\
2 a_{2}+a_{3}+a_{4}<1}} p_{1}\left(a_{1}+a_{3}, a_{1}+a_{4}, 1\right) p_{2}\left(a_{2}+a_{4}, a_{2}+a_{3}, 1\right) \mathrm{d} a_{1} \mathrm{~d} a_{2} \mathrm{~d} a_{3} \mathrm{~d} a_{4} \\
& +O_{p_{1}, p_{2}}\left((\operatorname{deg} Q)^{d_{1}+d_{2}+3}\right) \text {. }
\end{aligned}
$$

The proof of Lemma 7.8 is similar to the proof of Lemma 7.7. From [3], we use Lemma 7.10 instead of Lemma 7.9.

We can similarly prove the following:

Lemma 7.9 Let $Q \in \mathcal{P}$, let $p_{1}(\operatorname{deg} A, \operatorname{deg} B, \operatorname{deg} Q)$ and $p_{2}(\operatorname{deg} C, \operatorname{deg} D, \operatorname{deg} Q)$ be finite homogeneous polynomials of degree $d_{1}$ and $d_{2}$, respectively, and let $a \in\{0,1,2\}$. Then,

$$
\begin{aligned}
& \frac{1}{\phi(Q)} \sum_{\substack{\chi \bmod _{\chi} Q \\
\chi \neq \chi_{0}}}\left(\sum_{\substack{A, B \in \mathcal{M} \\
\operatorname{deg} A B=\operatorname{deg} Q-a}} \frac{p_{1} \chi(A) \bar{\chi}(B)}{|A B|^{\frac{1}{2}}}\right)\left(\sum_{\substack{C, D \in \mathcal{M} \\
\operatorname{deg} C D=\operatorname{deg} Q-a}} \frac{p_{2} \chi(C) \bar{\chi}(D)}{|C D|^{\frac{1}{2}}}\right) \\
& =O_{p_{1}, p_{2}}\left((\operatorname{deg} Q)^{d_{1}+d_{2}+3}\right),
\end{aligned}
$$

and

$$
\begin{aligned}
& \frac{1}{\phi(Q)} \sum_{\substack{\chi \bmod _{\chi} \text { even } \\
\chi \neq \chi_{0}}}\left(\sum_{\substack{A, B \in \mathcal{M} \\
\operatorname{deg} A B=\operatorname{deg} Q-a}} \frac{p_{1} \chi(A) \bar{\chi}(B)}{|A B|^{\frac{1}{2}}}\right)\left(\sum_{\substack{C, D \in \mathcal{M} \\
\operatorname{deg} C D=\operatorname{deg} Q-a}} \frac{p_{2} \chi(C) \bar{\chi}(D)}{|C D|^{\frac{1}{2}}}\right) \\
& =O_{p_{1}, p_{2}}\left((\operatorname{deg} Q)^{d_{1}+d_{2}+3}\right) .
\end{aligned}
$$

\section{Fourth moments of derivatives}

We are now equipped to prove the fourth moment result.

Proof of Theorem 2.6 We have that

$$
\begin{aligned}
& \frac{1}{\phi(Q)} \sum_{\substack{\chi \bmod Q \\
\chi \neq \chi_{0}}}\left|L^{(k)}\left(\frac{1}{2}, \chi\right)\right|^{2}\left|L^{(l)}\left(\frac{1}{2}, \chi\right)\right|^{2} \\
& =\frac{1}{\phi(Q)} \sum_{\substack{\chi \bmod Q \\
\chi \text { odd }}}\left|L^{(k)}\left(\frac{1}{2}, \chi\right)\right|^{2}\left|L^{(l)}\left(\frac{1}{2}, \chi\right)\right|^{2}+\frac{1}{\phi(Q)} \sum_{\substack{x \bmod Q \\
\text { meven } \\
\chi \neq \chi_{0}}}\left|L^{(k)}\left(\frac{1}{2}, \chi\right)\right|^{2}\left|L^{(l)}\left(\frac{1}{2}, \chi\right)\right|^{2} .
\end{aligned}
$$


Using Lemma 6.2, we have, for the first term on the RHS, that

$$
\begin{aligned}
& \frac{1}{\phi(Q)} \frac{1}{(\log q)^{2 k+2 l}} \sum_{\substack{\chi \bmod Q \\
\chi \text { odd }}}\left|L^{(k)}\left(\frac{1}{2}, \chi\right)\right|^{2}\left|L^{(l)}\left(\frac{1}{2}, \chi\right)\right|^{2} \\
& =\frac{1}{\phi(Q)} \sum_{\substack{\chi \bmod Q \\
\chi \text { odd }}}\left(\sum_{\substack{A, B \in \mathcal{M} \\
\operatorname{deg} A B<\operatorname{deg} Q}} \frac{\left(f_{k}+g_{O, k}\right) \chi(A) \bar{\chi}(B)}{|A B|^{\frac{1}{2}}}+\sum_{\substack{A, B \in \mathcal{M} \\
\operatorname{deg} A B=\operatorname{deg} Q-1}} \frac{h_{O, k} \chi(A) \bar{\chi}(B)}{|A B|^{\frac{1}{2}}}\right) \\
& \times\left(\sum_{\substack{C, D \in \mathcal{M} \\
\operatorname{deg} C D<\operatorname{deg} Q}} \frac{\left(f_{l}+g_{O, l}\right) \chi(C) \bar{\chi}(D)}{|C D|^{\frac{1}{2}}}+\sum_{\substack{C, D \in \mathcal{M} \\
\operatorname{deg} C D=\operatorname{deg} Q-1}} \frac{h_{O, l} \chi(C) \bar{\chi}(D)}{|C D|^{\frac{1}{2}}}\right) .
\end{aligned}
$$

By using Lemmas 7.7 and 7.8, we have that

$$
\begin{aligned}
& \frac{1}{\phi(Q)} \sum_{\substack{\chi \bmod Q \\
\chi \text { odd }}}\left(\sum_{\substack{A, B \in \mathcal{M} \\
\operatorname{deg} A B<\operatorname{deg} Q}} \frac{\left(f_{k}+g_{O, k}\right) \chi(A) \bar{\chi}(B)}{|A B|^{\frac{1}{2}}}\right)\left(\sum_{\substack{C, D \in \mathcal{M} \\
\operatorname{deg} C D<\operatorname{deg} Q}} \frac{\left(f_{l}+g_{O, l}\right) \chi(C) \bar{\chi}(D)}{|C D|^{\frac{1}{2}}}\right) \\
& =\frac{1}{\phi(Q)} \sum_{\substack{\chi \bmod _{\chi} Q \\
\chi \neq \chi_{0}}}\left(\sum_{\substack{A, B \in \mathcal{M} \\
\operatorname{deg} A B<\operatorname{deg} Q}} \frac{\left(f_{k}+g_{O, k}\right) \chi(A) \bar{\chi}(B)}{|A B|^{\frac{1}{2}}}\right)\left(\sum_{\substack{C, D \in \mathcal{M} \\
\operatorname{deg} C D<\operatorname{deg} Q}} \frac{\left(f_{l}+g_{O, l}\right) \chi(C) \bar{\chi}(D)}{|C D|^{\frac{1}{2}}}\right) \\
& -\frac{1}{\phi(Q)} \sum_{\substack{\chi \bmod _{\chi} \text { even } \\
\chi \neq \chi_{0}}}\left(\sum_{\substack{A, B \in \mathcal{M} \\
\operatorname{deg} A B<\operatorname{deg} Q}} \frac{\left(f_{k}+g_{O, k}\right) \chi(A) \bar{\chi}(B)}{|A B|^{\frac{1}{2}}}\right)\left(\sum_{\substack{C, D \in \mathcal{M} \\
\operatorname{deg} C D<\operatorname{deg} Q}} \frac{\left(f_{l}+g_{O, l}\right) \chi(C) \bar{\chi}(D)}{|C D|^{\frac{1}{2}}}\right) \\
& =\left(1-2 q^{-1}\right)(\operatorname{deg} Q)^{2 k+2 l+4} \\
& \times \int_{\substack{a_{1}, a_{2}, a_{3}, a_{4} \geq 0 \\
2 a_{2}+a_{3}+a_{4}<1}} f_{k}\left(a_{1}+a_{3}, a_{1}+a_{4}, 1\right) f_{l}\left(a_{2}+a_{4}, a_{2}+a_{3}, 1\right) \mathrm{d} a_{1} \mathrm{~d} a_{2} \mathrm{~d} a_{3} \mathrm{~d} a_{4} \\
& +O_{k, l}\left((\operatorname{deg} Q)^{2 k+2 l+3}\right) \text {. }
\end{aligned}
$$

Strictly speaking, Lemmas 7.7 and 7.8 require that the polynomials $f_{k}+g_{O, k}$ and $f_{l}+g_{O, l}$ are homogeneous, which is not the case. However, these polynomials can be written as sums of homogeneous polynomials, with the terms of highest degree being $f_{k}$ and $f_{l}$, respectively. We can then apply the lemmas term-by-term to obtain the result above.

We now have the main term of (16). Indeed, for the remaining terms we can apply the Cauchy-Schwarz inequality and Lemmas 7.7, 7.8, and 7.9 to see that they are equal to $O_{k, l}\left((\operatorname{deg} Q)^{2 k+2 l+\frac{7}{2}}\right)$. Hence,

$$
\begin{aligned}
& \frac{1}{\phi(Q)} \frac{1}{(\log q)^{2 k+2 l}} \sum_{\substack{\chi \bmod Q \\
\chi \text { odd }}}\left|L^{(k)}\left(\frac{1}{2}, \chi\right)\right|^{2}\left|L^{(l)}\left(\frac{1}{2}, \chi\right)\right|^{2} \\
& =\left(1-2 q^{-1}\right)(\operatorname{deg} Q)^{2 k+2 l+4} \\
& \quad \times \int_{\substack{a_{1}, a_{2}, a_{3}, a_{4} \geq 0 \\
2 a_{1}+a_{3}+a_{4}<1 \\
2 a_{2}+a_{3}+a_{4}<1}} f_{k}\left(a_{1}+a_{3}, a_{1}+a_{4}, 1\right) f_{l}\left(a_{2}+a_{4}, a_{2}+a_{3}, 1\right) \mathrm{d} a_{1} \mathrm{~d} a_{2} \mathrm{~d} a_{3} \mathrm{~d} a_{4} \\
& +O_{k, l}\left((\operatorname{deg} Q)^{2 k+2 l+\frac{7}{2}}\right) .
\end{aligned}
$$


We now look at the second term on the RHS of (15). By using Lemma 6.7 and similar means as those used to deduce (17), we can show for all non-negative integers $i, j$ that

$$
\begin{aligned}
& \frac{1}{\phi(Q)} \frac{1}{(\log q)^{2 i+2 j}} \frac{1}{\left(q^{\frac{1}{2}}-1\right)^{4}} \sum_{\substack{x \bmod Q \\
\chi \text { even } \\
\chi \neq \chi_{0}}}\left|\hat{L}^{(i)}\left(\frac{1}{2}, \chi\right)\right|^{2}\left|\hat{L}^{(j)}\left(\frac{1}{2}, \chi\right)\right|^{2} \\
& =q^{-1}(\operatorname{deg} Q)^{2 i+2 j+4} \quad \\
& \quad \times \int_{\substack{a_{1}, a_{2}, a_{3}, a_{4} \geq 0 \\
2 a_{1}+a_{3}+a_{4}<1 \\
2 a_{2}+a_{3}+a_{4}<1}} f_{i}\left(a_{1}+a_{3}, a_{1}+a_{4}, 1\right) f_{j}\left(a_{2}+a_{4}, a_{2}+a_{3}, 1\right) \mathrm{d} a_{1} \mathrm{~d} a_{2} \mathrm{~d} a_{3} \mathrm{~d} a_{4} \\
& +O_{i, j}\left((\operatorname{deg} Q)^{2 i+2 j+\frac{7}{2}}\right) .
\end{aligned}
$$

Using Lemma 6.5 and the Cauchy-Schwarz inequality, we obtain that

$$
\begin{aligned}
& \frac{1}{\phi(Q)} \frac{1}{(\log q)^{2 k+2 l}} \sum_{\substack{x \text { mod } Q \\
\chi \text { even } \\
\chi \neq \chi_{0}}}\left|L^{(k)}\left(\frac{1}{2}, \chi\right)\right|^{2}\left|L^{(l)}\left(\frac{1}{2}, \chi\right)\right|^{2} \\
& =q^{-1}(\operatorname{deg} Q)^{2 k+2 l+4} \times \int_{\substack{a_{1}, a_{2}, a_{3}, a_{4} \geq 0 \\
2 a_{2}+a_{3}+a_{4}<1 \\
a_{2}+a_{3}+a_{4}<1}} f_{k}\left(a_{1}+a_{3}, a_{1}+a_{4}, 1\right) f_{l}\left(a_{2}+a_{4}, a_{2}+a_{3}, 1\right) \mathrm{d} a_{1} \mathrm{~d} a_{2} \mathrm{~d} a_{3} \mathrm{~d} a_{4} \\
& \quad+O_{k, l}\left((\operatorname{deg} Q)^{2 k+2 l+\frac{7}{2}}\right) .
\end{aligned}
$$

The proof follows from (15), (17), (18).

We now proceed to prove Theorem 2.7.

Lemma 8.1 Let $m$ be a positive integer. For all non-negative $x$ we have that

$$
\left(1-\frac{x}{m}\right)^{m} \leq e^{-x}
$$

and for all $x \in\left[0,2 m^{\frac{1}{3}}\right]$ we have that

$$
\left(1-\frac{x}{m}\right)^{m} \geq e^{-x} e^{\frac{-4}{m^{\frac{1}{3}}-2 m^{-\frac{1}{3}}}} .
$$

Proof By using the Taylor series for log we have that

$$
\log \left(\left(1-\frac{x}{m}\right)^{m}\right)=-x-\frac{x^{2}}{2 m}-\frac{x^{3}}{3 m^{2}}-\frac{x^{4}}{4 m^{3}}-\ldots
$$

Clearly, the RHS is $\leq-x$, which proves the first inequality. For the second inequality we use the bounds on $x$ to obtain that

$$
\frac{x^{2}}{2 m}+\frac{x^{3}}{3 m^{2}}+\frac{x^{4}}{4 m^{3}}+\ldots \leq \frac{x^{2}}{m} \sum_{i=0}^{\infty}\left(\frac{x}{m}\right)^{i}=\frac{x^{2}}{m}\left(\frac{1}{1-\frac{x}{m}}\right) \leq\left(\frac{4}{m^{\frac{1}{3}}-2 m^{-\frac{1}{3}}}\right),
$$

from which the result follows.

Proof of Theorem 2.7 Let us expand the brackets in (7) and multiply by $m^{4}$. One of the terms is the following: 


$$
\begin{gathered}
m^{4} \int_{\substack{a_{1}, a_{2}, a_{3}, a_{4} \geq 0 \\
2 a_{1}+a_{3}+a_{4}<1 \\
2 a_{2}+a_{3}+a_{4}<1}}\left(1-a_{1}-a_{3}\right)^{m}\left(1-a_{1}-a_{4}\right)^{m}\left(1-a_{2}-a_{3}\right)^{m}\left(1-a_{2}-a_{4}\right)^{m} \mathrm{~d} a_{1} \mathrm{~d} a_{2} \mathrm{~d} a_{3} \mathrm{~d} a_{4} \\
=\int_{\substack{a_{1}, a_{2}, a_{3}, a_{4} \geq 0 \\
2 a_{1}+a_{3}+a_{4}<m \\
2 a_{2}+a_{3}+a_{4}<m}}\left(1-\frac{a_{1}+a_{3}}{m}\right)^{m}\left(1-\frac{a_{1}+a_{4}}{m}\right)^{m} \\
\times\left(1-\frac{a_{2}+a_{3}}{m}\right)^{m}\left(1-\frac{a_{2}+a_{4}}{m}\right)^{m} \mathrm{~d} a_{1} \mathrm{~d} a_{2} \mathrm{~d} a_{3} \mathrm{~d} a_{4},
\end{gathered}
$$

where we have used the substitutions $a_{i} \rightarrow \frac{a_{i}}{m}$. On one hand, by using Lemma 8.1, we have that

$$
\begin{aligned}
& \int_{\substack{a_{1}, a_{2}, a_{3}, a_{4} \geq 0 \\
2 a_{1}+a_{3}+a_{4}<m \\
2 a_{2}+a_{3}+a_{4}<m}}\left(1-\frac{a_{1}+a_{3}}{m}\right)^{m}\left(1-\frac{a_{1}+a_{4}}{m}\right)^{m}\left(1-\frac{a_{2}+a_{3}}{m}\right)^{m} \\
& \times\left(1-\frac{a_{2}+a_{4}}{m}\right)^{m} \mathrm{~d} a_{1} \mathrm{~d} a_{2} \mathrm{~d} a_{3} \mathrm{~d} a_{4} \\
& \geq \int_{\substack{0 \leq a_{1}, a_{2}, a_{3}, a_{4} \leq m \\
2 a_{1}+a_{3}+a_{4}<m \\
2 a_{2}+a_{3}+a_{4}<m}} \frac{1}{3}\left(1-\frac{a_{1}+a_{3}}{m}\right)^{m}\left(1-\frac{a_{1}+a_{4}}{m}\right)^{m} \\
& \times\left(1-\frac{a_{2}+a_{3}}{m}\right)^{m}\left(1-\frac{a_{2}+a_{4}}{m}\right)^{m} \mathrm{~d} a_{1} \mathrm{~d} a_{2} \mathrm{~d} a_{3} \mathrm{~d} a_{4} \\
& \geq e^{\frac{-16}{m^{\frac{1}{3}}-2 m^{-\frac{1}{3}}}} \int_{0 \leq a_{1}, a_{2}, a_{3}, a_{4} \leq m^{\frac{1}{3}}} e^{-2\left(a_{1}+a_{2}+a_{3}+a_{4}\right)} \mathrm{d} a_{1} \mathrm{~d} a_{2} \mathrm{~d} a_{3} \mathrm{~d} a_{4} \longrightarrow \frac{1}{16}
\end{aligned}
$$

as $m \longrightarrow \infty$. On the other hand, by the same lemma, we have that

$$
\begin{gathered}
\int_{\substack{a_{1}, a_{2}, a_{3}, a_{4} \geq 0 \\
2 a_{1}+a_{3}+a_{4}<m \\
2 a_{2}+a_{3}+a_{4}<m}}\left(1-\frac{a_{1}+a_{3}}{m}\right)^{m}\left(1-\frac{a_{1}+a_{4}}{m}\right)^{m}\left(1-\frac{a_{2}+a_{3}}{m}\right)^{m} \\
\times\left(1-\frac{a_{2}+a_{4}}{m}\right)^{m} \mathrm{~d} a_{1} \mathrm{~d} a_{2} \mathrm{~d} a_{3} \mathrm{~d} a_{4} \\
\leq \int_{0 \leq a_{1}, a_{2}, a_{3}, a_{4} \leq m} e^{-2\left(a_{1}+a_{2}+a_{3}+a_{4}\right)} \mathrm{d} a_{1} \mathrm{~d} a_{2} \mathrm{~d} a_{3} \mathrm{~d} a_{4} \rightarrow \frac{1}{16}
\end{gathered}
$$

as $m \longrightarrow \infty$. So, we see that

$$
\begin{aligned}
& m^{4} \int_{\substack{a_{1}, a_{2}, a_{3}, a_{4} \geq 0 \\
2 a_{1}+a_{3}+a_{4}<1 \\
2 a_{2}+a_{3}+a_{4}<1}}\left(1-a_{1}-a_{3}\right)^{m}\left(1-a_{1}-a_{4}\right)^{m}\left(1-a_{2}-a_{3}\right)^{m}\left(1-a_{2}-a_{4}\right)^{m} \mathrm{~d} a_{1} \mathrm{~d} a_{2} \mathrm{~d} a_{3} \mathrm{~d} a_{4} \\
& \quad \longrightarrow \frac{1}{16}
\end{aligned}
$$

as $m \longrightarrow \infty$.

Now, after we expanded the brackets in (7) and multiplied by $m^{4}$, there were other terms. These can be seen to tend to 0 as $m \longrightarrow \infty$. We prove one case below; the rest are similar.

$$
\begin{aligned}
& m^{4} \int_{\substack{a_{1}, a_{2}, a_{3}, a_{4} \geq 0 \\
2 a_{1}+a_{3}+a_{4}<1 \\
2 a_{2}+a_{3}+a_{4}<1}}\left(1-a_{1}-a_{3}\right)^{m}\left(1-a_{1}-a_{4}\right)^{m}\left(a_{2}+a_{3}\right)^{m}\left(a_{2}+a_{4}\right)^{m} \mathrm{~d} a_{1} \mathrm{~d} a_{2} \mathrm{~d} a_{3} \mathrm{~d} a_{4} \\
& \leq m^{4} \int_{\substack{a_{1}, a_{2}, a_{3}, a_{4} \geq 0 \\
2 a_{1}+a_{3}+a_{4}<1 \\
2 a_{2}+a_{3}+a_{4}<1}}\left(a_{2}+a_{3}\right)^{m}\left(a_{2}+a_{4}\right)^{m} \ll \frac{m^{4}}{4^{m}},
\end{aligned}
$$


where we have used the following: The maximum value that $\left(a_{2}+a_{3}\right)\left(a_{2}+a_{4}\right)$ can take subject to the conditions in the integral is at most equal to the maximum value that $f(x, y):=x y$ can take subject to the conditions $x, y \geq 0$ and $x+y<1$. By plotting this range and looking at contours of $f(x, y)$ we can see that the maximum value is $\frac{1}{4}$. The result follows.

Acknowledgements The first author is grateful to the Leverhulme Trust (RPG-2017-320) for the support through the research project grant "Moments of $L$-functions in Function Fields and Random Matrix Theory". The second author is grateful for an EPSRC Standard Research Studentship (DTP) (EP/M506527/1). Both authors are grateful to Nigel Byott and Hung Bui for pointing out errors and making suggestions for improvements.

Open Access This article is licensed under a Creative Commons Attribution 4.0 International License, which permits use, sharing, adaptation, distribution and reproduction in any medium or format, as long as you give appropriate credit to the original author(s) and the source, provide a link to the Creative Commons licence, and indicate if changes were made. The images or other third party material in this article are included in the article's Creative Commons licence, unless indicated otherwise in a credit line to the material. If material is not included in the article's Creative Commons licence and your intended use is not permitted by statutory regulation or exceeds the permitted use, you will need to obtain permission directly from the copyright holder. To view a copy of this licence, visit http://creativecommons.org/licenses/by/4.0/.

\section{References}

1. Andrade, J.C., Keating, J.P.: The mean value of $L\left(\frac{1}{2}, \chi\right)$ in the hyperelliptic ensemble. J. Number Theory 132, 2793-2816 (2012)

2. Andrade, J.C., Keating, J.P.: Mean value theorems for $L$-functions over prime polynomials for the rational function field. Acta Arith. 161(4), 371-385 (2013)

3. Andrade, J.C., Yiasemides, M.: The fourth power mean of Dirichlet $L$-functions in $\mathbb{F}_{q}[T]$. Rev. Mat. Complut. https://doi.org/10.1007/s13163-020-00350-2

4. Bui, H.M., Conrey, B., Young, M.P.: More than $41 \%$ of the zeros of the zeta function are on the critical line. Acta Arith. 150(1), 35-64 (2011)

5. Bui, H.M., Florea, A.: Hybrid Euler-Hadamard product for quadratic Dirichlet $L$-functions in function fields. Proc. Lond. Math. Soc. 117(3), 65-99 (2018)

6. Bui, H.M., Keating, J.P.: On the mean values of Dirichlet $L$-functions. Proc. Lond. Math. Soc. 95(2), 273-298 (2007)

7. Conrey, J.B., Farmer, D.W.: Mean values of $L$-functions and symmetry. Int. Math. Res. Not. 2000(17), 883-908 (2000)

8. Conrey, J.B.: The fourth moment of derivatives of the Riemann zeta-function. Q. J. Math. 39(1), 21-36 (1988)

9. Conrey, J.B.: More than two fifths of the zeros of the Riemann zeta function are on the critical line. J. Reine Angew. Math. 399, 1-26 (1989)

10. Conrey, J.B., Rubinstein, M.O., Snaith, N.C.: Moments of the derivative of characteristic polynomials with an application to the Riemann zeta function. Commun. Math. Phys. 267(3), 611-629 (2006)

11. Gonek, S.M., Hughes, C.P., Keating, J.P.: A hybrid Euler-Hadamard product for the Riemann zeta function. Duke Math. J. 136(3), 507-549 (2007)

12. Gonek, S.M.: Applications of mean value theorems to the theory of the Riemann zeta function; page 201224; London Mathematical Society Lecture Note Series. Cambridge University Press (2005). https:// doi.org/10.1017/CBO9780511550492.008

13. Hardy, G.H., Littlewood, J.E.: Contributions to the theory of the Riemann zeta-function and the distribution of primes. Acta Math. 41, 119-196 (1916)

14. Heath-Brown, D.R.: The fourth power moment of the Riemann zeta function. Proc. Lond. Math. Soc. 38(3), 385-422 (1979)

15. Heath-Brown, D.R.: The fourth power mean of Dirichlet's $L$-functions. Analysis 1(1), 25-32 (1981)

16. Ingham, A.E.: Mean-value theorems in the theory of the Riemann zeta-function. Proc. Lond. Math. Soc. 27, 273-300 (1926)

17. Katz, N.M., Sarnak, P.: Zeroes of zeta functions and symmetry. Bull. Am. Math. Soc. 36(1), 1-26 (1999)

18. Keating, J.P., Snaith, N.C.: Random matrix theory and $\zeta\left(\frac{1}{2}+i t\right)$. Commun. Math. Phys. 214, 57-89 (2000) 
19. Lang, S.: Algebra, 3rd edn. Springer, New York (2002)

20. Levinson, N.: More than one third of zeros of Riemann's zeta function are on $\sigma=1 / 2$. Adv. Math. 13, 383-436 (1974)

21. Liu, S.: On central $L$-derivative values of automorphic forms. Math. Z. 288, 1327-1359 (2018)

22. Paley, E.A.C.: On the $k$-analogues of some theorems in the theory of the Riemann zeta-function. Proc. Lond. Math. Soc. S2-32, 273-311 (1931)

23. Rosen, M.: Number Theory in Function Fields. Springer, New York (2002)

24. Soundararajan, K.: The fourth moment of Dirichlet $L$-functions. Clay Math. Proc. 7, 239-246 (2007)

25. Tamam, N.: The fourth moment of Dirichlet $L$-functions for the rational function field. Int. J. Number Theory 10(1), 183-218 (2014)

26. Young, M.P.: The fourth moment of Dirichlet L-functions. Ann. Math. 173, 1-50 (2011)

Publisher's Note Springer Nature remains neutral with regard to jurisdictional claims in published maps and institutional affiliations. 